\title{
Prym varieties of spectral covers
}

\author{
TAMÁS HAUSEL \\ Christian PAUly
}

\begin{abstract}
Given a possibly reducible and non-reduced spectral cover $\pi: X \rightarrow C$ over a smooth projective complex curve $C$ we determine the group of connected components of the Prym variety $\operatorname{Prym}(X / C)$. As an immediate application we show that the finite group of $n$-torsion points of the Jacobian of $C$ acts trivially on the cohomology of the twisted $\mathrm{SL}_{n}$-Higgs moduli space up to the degree which is predicted by topological mirror symmetry. In particular this yields a new proof of a result of Harder-Narasimhan, showing that this finite group acts trivially on the cohomology of the twisted $\mathrm{SL}_{n}$ stable bundle moduli space.
\end{abstract}

14K30; 14H60, 14H40

\section{Introduction}

Recently there has been renewed interest in the topology of the Hitchin fibration. The Hitchin fibration is an integrable system associated to a complex reductive group $\mathrm{G}$ and a smooth complex projective curve $C$. It was introduced by Hitchin [18] in 1987, originating in his study of a 2-dimensional reduction of the Yang-Mills equations. In 2006, Kapustin and Witten [20] highlighted the importance of the Hitchin fibration for $S$-duality and the Geometric Langlands program. While the work of Ngô [28] in 2008 showed that the topology of the Hitchin fibration is responsible for the fundamental lemma in the Langlands program. In Ngô's work and later in the work of Frenkel and Witten [9] a certain symmetry of the Hitchin fibration plays an important role.

In this paper we focus on the Hitchin fibration for the group $\mathrm{G}=\mathrm{SL}_{n}$ and for a line bundle $M$ over $C$, that is, the morphism

$$
h: \mathcal{M} \longrightarrow \mathcal{A}_{n}^{0}=\bigoplus_{j=2}^{n} H^{0}\left(C, M^{j}\right) .
$$

Here $\mathcal{M}$ denotes the quasi-projective moduli space of semi-stable Higgs bundles $(E, \phi)$ over $C$ of rank $n$, fixed determinant $\Delta$ and with trace-free Higgs field $\phi \in$ $H^{0}\left(C, \operatorname{End}_{0}(E) \otimes M\right)$. In the case of $\mathrm{SL}_{n}$ the above mentioned symmetry group 
of the Hitchin fibration is the Prym variety of a spectral cover. For the topological applications the determination of its group of components is the first step. Ngô works with integral, that is irreducible and reduced, spectral curves; but it is interesting to extend his results to non-integral curves. For reducible but reduced spectral curves it was achieved by Chaudouard and Laumon [5], who proved the weighted fundamental lemma by generalizing Ngô's results to reduced spectral curves. In this paper we determine the group of connected components of the Prym variety for non-reduced spectral curves as well.

In order to state the main theorem we need to introduce some notation. We associate to any spectral cover $\pi: X \rightarrow C$ a finite group $K$ as follows: let $X=\bigcup_{i \in I} X_{i}$ be its decomposition into irreducible components $X_{i}$, let $X_{i}^{\text {red }}$ be the underlying reduced curve of $X_{i}, m_{i}$ the multiplicity of $X_{i}^{\text {red }}$ in $X_{i}$ and $\tilde{X}_{i}^{\text {red }}$ the normalization of $X_{i}$. We denote by $\tilde{\pi}_{i}: \tilde{X}_{i}^{\text {red }} \rightarrow C$ the projection onto $C$ and introduce the finite subgroups

$$
K_{i}=\operatorname{ker}\left(\tilde{\pi}_{i}^{*}: \operatorname{Pic}^{0}(C) \longrightarrow \operatorname{Pic}^{0}\left(\tilde{X}_{i}^{\text {red }}\right)\right) \subset \operatorname{Pic}^{0}(C),
$$

as well as the subgroups $\left(K_{i}\right)_{m_{i}}=\left[m_{i}\right]^{-1}\left(K_{i}\right)$, where $\left[m_{i}\right]$ denotes multiplication by $m_{i}$ in the Picard variety $\operatorname{Pic}^{0}(C)$ parameterizing degree 0 line bundles over $C$. Finally, we put

$$
K=\bigcap_{i \in I}\left(K_{i}\right)_{m_{i}} \subset \operatorname{Pic}^{0}(C)
$$

We denote by $C_{n}$ the multiple curve with trivial nilpotent structure of order $n$ having underlying reduced curve $C$.

We consider the norm map $\mathrm{Nm}_{X / C}$ : $\operatorname{Pic}^{0}(X) \rightarrow \operatorname{Pic}^{0}(C)$ between the connected components of the identity elements of the Picard schemes of the curves $X$ and $C$ and define the Prym variety

$$
\operatorname{Prym}(X / C):=\operatorname{ker}\left(\mathrm{Nm}_{X / C}\right) .
$$

Our main result is the following

Theorem 1.1 Let $\pi: X \rightarrow C$ be a spectral cover of degree $n \geq 2$. With the notation above we have the following results:

(1) The group of connected components $\pi_{0}(\operatorname{Prym}(X / C))$ of the Prym variety $\operatorname{Prym}(X / C)$ equals

$$
\pi_{0}(\operatorname{Prym}(X / C))=\widehat{K},
$$

where $\hat{K}=\operatorname{Hom}\left(K, \mathbb{C}^{*}\right)$ is the group of characters of $K$. 
(2) The natural homomorphism from the group of $n$-torsion line bundles $\operatorname{Pic}^{0}(C)[n]$ to $\pi_{0}(\operatorname{Prym}(X / C))$ given by

$$
\Phi: \operatorname{Pic}^{0}(C)[n] \longrightarrow \pi_{0}(\operatorname{Prym}(X / C)), \quad \gamma \mapsto\left[\pi^{*} \gamma\right],
$$

where $\left[\pi^{*} \gamma\right]$ denotes the class of $\pi^{*} \gamma \in \operatorname{Pic}^{0}(X)$ in $\pi_{0}(\operatorname{Prym}(X / C))$ is surjective. In particular, we obtain an upper bound for the order

$$
\left|\pi_{0}(\operatorname{Prym}(X / C))\right| \leq n^{2 g},
$$

where $g$ is the genus of the curve $C$.

(3) The map $\Phi$ is an isomorphism if and only if $X$ equals the non-reduced curve $C_{n}$ with trivial nilpotent structure of order $n$.

Similar descriptions of $\pi_{0}(\operatorname{Prym}(X / C))$ were given by $\mathrm{Ngô}$ in [27] in the case of integral spectral curves and by Chaudouard and Laumon [5] in the case of reducible but reduced spectral curves. Also de Cataldo, Hausel and Migliorini [6] use special cases for $\mathrm{SL}_{2}$.

For a characteristic $a \in \mathcal{A}_{n}^{0}$ we denote by $\pi: X_{a} \rightarrow C$ the associated spectral cover of degree $n$ (see Section 2.2) and by $K_{a}$ the subgroup of $\operatorname{Pic}^{0}(C)$ defined in (2) and corresponding to the cover $X_{a}$. Let $\Gamma \subset \operatorname{Pic}^{0}(C)[n]$ be a cyclic subgroup of order $d$ of the finite group $\operatorname{Pic}^{0}(C)[n]$ of $n$-torsion line bundles over $C$ and let $\mathcal{A}_{\Gamma}^{0} \subset \mathcal{A}_{n}^{0}$ denote the endoscopic sublocus of characteristics $a$ such that the associated degree $n$ spectral cover $\pi: X_{a} \rightarrow C$ comes from a degree $\frac{n}{d}$ spectral cover over the étale Galois cover of $C$ with Galois group $\Gamma$ (for the precise definition see Section 5.1). With this notation we have the following

Theorem 1.2 We have an equivalence

$$
\Gamma \subset K_{a} \quad \Longleftrightarrow \quad a \in \mathcal{A}_{\Gamma}^{0} .
$$

This gives a description of the locus of characteristics $a \in \mathcal{A}_{n}^{0}$ such that the Prym variety $\operatorname{Prym}\left(X_{a} / C\right)$ is non-connected, because clearly $\mathcal{A}_{\Gamma_{2}}^{0} \subset \mathcal{A}_{\Gamma_{1}}^{0}$ if $\Gamma_{1} \subset \Gamma_{2}$.

Corollary 1.3 The sublocus of characteristics $a \in \mathcal{A}_{n}^{0}$ such that the Prym variety $\operatorname{Prym}\left(X_{a} / C\right)$ is not connected equals the union

$$
\mathcal{A}_{\text {endo }}:=\bigcup \mathcal{A}_{\Gamma}^{0},
$$

where $\Gamma$ varies over all cyclic subgroups of prime order of $\operatorname{Pic}^{0}(C)[n]$. 
Calculating the dimensions of the endoscopic loci $\mathcal{A}_{\Gamma}^{0}$ will lead to an immediate topological application. Recall that $\operatorname{Pic}^{0}(C)[n]$ acts on $\mathcal{M}$ by tensorization, and this will induce an action on the rational cohomology $H^{*}(\mathcal{M} ; \mathbb{Q})$. We then have

Theorem 1.4 Let $n>1$ and $p_{n}$ be the smallest prime divisor of $n$. Assume that $M=K_{C}$, the canonical bundle of $C$, and that $(n, \operatorname{deg}(\Delta))=1$. Then the action of $\operatorname{Pic}^{0}(C)[n]$ on $H^{k}(\mathcal{M} ; \mathbb{Q})$ is trivial, provided that

$$
k \leq 2 n^{2}\left(1-1 / p_{n}\right)(g-1) .
$$

In fact this result should be sharp, as the topological mirror symmetry conjecture of Hausel and Thaddeus [17, Conjecture 5.1] predicts that the smallest degree where $\operatorname{Pic}^{0}(C)[n]$ acts non-trivially is

$$
k=n^{2}\left(1-1 / p_{n}\right)(2 g-2)+1 .
$$

This results hints at the close connection between Ngô's strategy in [27; 28] for studying the symmetries of the Hitchin fibration and the topological mirror symmetry conjectures in [17]. More discussion on this connection can be found in Hausel [15].

Finally let $\mathcal{N}$ denote the moduli space of stable vector bundles of rank $n$ and fixed determinant $\Delta$ over $C$. Again the finite group $\operatorname{Pic}^{0}(C)[n]$ acts on $\mathcal{N}$ by tensorization and thus on $H^{*}(\mathcal{N} ; \mathbb{Q})$. As the cohomology $H^{*}(\mathcal{N} ; \mathbb{Q})$ is a summand in the cohomology of $H^{*}(\mathcal{M} ; \mathbb{Q})$ we can deduce

Corollary 1.5 The finite group $\operatorname{Pic}^{0}(C)[n]$ acts trivially on $H^{*}(\mathcal{N} ; \mathbb{Q})$.

This was the main application of Harder-Narasimhan in [13, Theorem 1]. Our proof here can be considered as an example of both the abelianization philosophy of AtiyahHitchin [1, Section 6.3] and Ngô's strategy [27; 28] of studying the symmetries of the Hitchin fibration.

The paper is organized as follows. In sections 2 and 3 we recall basic results on spectral covers and on the norm map $\mathrm{Nm}_{X / C}$. In sections 4 and 5 we prove the two main theorems. In section 6 we describe the action of the Prym variety $\operatorname{Prym}\left(X_{a} / C\right)$ on the fiber over $a \in \mathcal{A}_{n}^{0}$ of the $\mathrm{SL}_{n}-$ Hitchin fibration. Finally in section 7 we apply the results in this paper to prove Theorem 1.4 and its Corollary 1.5.

Notation Given a sheaf $\mathcal{F}$ over a scheme $X$ and a subset $U \subset X$ we denote by $\mathcal{F}(U)$ or by $\Gamma(U, \mathcal{F})$ the space of sections of $\mathcal{F}$ over $U$. 
Acknowledgements During the preparation of this paper the first author was supported by a Royal Society University Research Fellowship while the second author was supported by a Marie Curie Intra-European Fellowship (PIEF-GA-2009-235098) from the European Commission. We would like to thank Jean-Marc Drézet, Nigel Hitchin, M S Narasimhan and Daniel Schaub for helpful discussions.

\section{Preliminaries}

\subsection{Two lemmas on abelian varieties}

Given an abelian variety $A$ and a positive integer $n$ we denote by $[n]: A \rightarrow A$ the multiplication by $n$, by $A[n]=\operatorname{ker}[n]$ its subgroup of $n$-torsion points and by $\hat{A}=$ $\operatorname{Pic}^{0}(A)$ its dual abelian variety. We consider

$$
f: A \longrightarrow B
$$

a homomorphism between abelian varieties with kernel $K=\operatorname{ker}(f)$ which we assume to be finite. We let $\hat{f}: \hat{B} \rightarrow \widehat{A}$ denote the dual map induced by $f$. We introduce the quotient abelian variety $A^{\prime}=A / K$, so that we can write the homomorphism $f$ as a composite map

$$
f=j \circ \mu: A \stackrel{\mu}{\longrightarrow} A^{\prime} \stackrel{j}{\longrightarrow} B,
$$

where $\mu$ is an isogeny with kernel $K$ and $j$ is injective.

Lemma 2.1 The group of connected components of the abelian subvariety $\operatorname{ker}(\widehat{f}) \subset \widehat{B}$ equals

$$
\pi_{0}(\operatorname{ker}(\widehat{f}))=\widehat{K}
$$

where $\widehat{K}=\operatorname{Hom}\left(K, \mathbb{C}^{*}\right)$ is the group of characters of $K$.

Proof We consider the dual map

$$
\widehat{f}: \hat{B} \stackrel{\hat{j}}{\longrightarrow} \widehat{A}^{\prime} \stackrel{\widehat{\mu}}{\longrightarrow} \widehat{A},
$$

and observe that $\widehat{\mu}: \widehat{A}^{\prime} \rightarrow \widehat{A}$ is an isogeny with kernel $\widehat{K}$ (see, for example, Birkenhake and Lange [4, Proposition 2.4.3]) and $\hat{j}$ has connected fibers (see, for example, [4, Proposition 2.4.2]). The lemma then follows.

We also suppose that $A$ and $B$ are principally polarized abelian varieties, that is, the polarizations induce isomorphisms $A \cong \widehat{A}$ and $B \cong \widehat{B}$. 
Lemma 2.2 We assume that there exists a homomorphism $g: B \rightarrow A$ such that $g \circ f=[n]$ for some integer $n$. Then the dual of the canonical inclusion $i: K \hookrightarrow A[n]$ is a surjective map

$$
\hat{i}: A[n]=\widehat{A}[n] \longrightarrow \widehat{K},
$$

which coincides with the restriction to $A[n]$ of the composite map $\hat{j} \circ \hat{g}: A \rightarrow B \rightarrow \widehat{A}^{\prime}$.

Proof It suffices to observe that the isogeny $\widehat{f} \circ \widehat{g}=[\widehat{n}]=[n]$ factorizes as

$$
[n]: A \stackrel{\hat{j} \circ \hat{g}}{\longrightarrow} \widehat{A}^{\prime} \stackrel{\widehat{\mu}}{\longrightarrow} A,
$$

that $\hat{K}=\operatorname{ker}(\hat{\mu})$, and that $\hat{j} \circ \hat{g}$ is surjective. Hence a canonical surjection $A[n] \rightarrow \widehat{K}$, which is dual to the inclusion $i: K \hookrightarrow A[n]$, since $[\widehat{n}]=[n]$.

\subsection{Spectral covers}

In this section we review some elementary facts on spectral covers.

Let $C$ be a complex smooth projective curve and let $M$ be a line bundle over $C$ with $\operatorname{deg} M>0$. We denote by $|M|$ the total space of $M$ and by

$$
\pi:|M| \longrightarrow C
$$

the projection onto $C$. There is a canonical coordinate $t \in H^{0}\left(|M|, \pi^{*} M\right)$ on the total space $|M|$. The direct image decomposes as follows

$$
\pi_{*} \mathcal{O}_{|M|}=\operatorname{Sym}^{\bullet}\left(M^{-1}\right)=\bigoplus_{i=0}^{\infty} M^{-i}
$$

Definition 2.3 A spectral cover $X$ of degree $n$ over the curve $C$ and associated to the line bundle $M$ is the zero divisor in $|M|$ of a non-zero section $s \in H^{0}\left(|M|, \pi^{*} M^{n}\right)$.

Since a spectral cover $X$ is a subscheme of $|M|$, it is naturally equipped with a projection onto $C$, which we also denote by $\pi$. The decomposition of the section $s$ according to the direct sum

$$
\begin{aligned}
H^{0}\left(|M|, \pi^{*} M^{n}\right) & =H^{0}\left(C, M^{n} \otimes \bigoplus_{i=0}^{\infty} M^{-i}\right) \quad \text { (projection formula) } \\
& =H^{0}\left(C, M^{n}\right) \oplus \cdots \oplus H^{0}(C, M) \oplus H^{0}\left(C, \mathcal{O}_{C}\right)
\end{aligned}
$$

gives an expression $s=s_{0}+t s_{1}+\cdots+t^{n-1} s_{n-1}+t^{n} s_{n}$ with $s_{j} \in H^{0}\left(C, M^{n-j}\right)$. Here we also denote by $s_{j}$ its pull-back to $|M|$. We note that there is an isomorphism 
$\pi^{*}: \operatorname{Pic}(C) \stackrel{\sim}{\longrightarrow} \operatorname{Pic}(|M|)$, hence any line bundle over $|M|$ is of the form $\pi^{*} L$ for some line bundle $L \in \operatorname{Pic}(C)$. More generally, we have a decomposition

$$
H^{0}\left(|M|, \pi^{*} L\right)=H^{0}(C, L) \oplus H^{0}\left(C, L M^{-1}\right) \oplus \cdots \oplus H^{0}\left(C, L M^{-d}\right)
$$

for some integer $d$ and any section $s \in H^{0}\left(|M|, \pi^{*} L\right)$ can be written in the form

$$
s=s_{0}+t s_{1}+\cdots+t^{d-1} s_{d-1}+t^{d} s_{d}, \quad s_{j} \in H^{0}\left(C, L M^{-j}\right) .
$$

Lemma 2.4 Let $\pi: X \rightarrow C$ be a spectral cover. Then the underlying reduced curve of each irreducible component of $X$ is again a spectral cover associated to the line bundle $M$.

Proof It suffices to show that if the section $s \in H^{0}\left(|M|, \pi^{*} M^{n}\right)$ decomposes as $s=s^{(1)} \cdot s^{(2)}$ with $s^{(i)} \in H^{0}\left(|M|, \pi^{*} L_{i}\right)$ for $i=1,2$ and $L_{1} L_{2}=M^{n}$, then $L_{i}=M^{n_{i}}$ and $n_{1}+n_{2}=n$. By (4) the section $s^{(i)}$ can be written as

$$
s^{(i)}=s_{0}^{(i)}+t s_{1}^{(i)}+\cdots+t^{n_{i}} s_{n_{i}}^{(i)},
$$

with $s_{j}^{(i)} \in H^{0}\left(C, L_{i} M^{-j}\right)$ and $s_{n_{i}}^{(i)} \neq 0$. Moreover $n_{i}=\operatorname{deg}\left(X^{(i)} / C\right)$ with $X^{(i)}=$ Zeros $\left(s^{(i)}\right)$. By considering the highest order terms of (5) we obtain the relations $n_{1}+n_{2}=n$ and $s_{n_{1}}^{(1)} \cdot s_{n_{2}}^{(2)}=s_{n} \in H^{0}\left(C, \mathcal{O}_{C}\right)$. Since $s_{n}$ is a non-zero constant section, we conclude that $L_{i}=M^{n_{i}}$.

We introduce the $\mathrm{SL}_{n}$ - and $\mathrm{GL}_{n}$-Hitchin space for the line bundle $M$ over the curve C

$$
\mathcal{A}_{n}^{0}(C, M)=\bigoplus_{j=2}^{n} H^{0}\left(C, M^{j}\right) \text { and } \mathcal{A}_{n}(C, M)=\bigoplus_{j=1}^{n} H^{0}\left(C, M^{j}\right) .
$$

If no confusion arises, we simply denote these vector spaces by $\mathcal{A}_{n}^{0}$ and $\mathcal{A}_{n}$. Note that $\mathcal{A}_{n}^{0} \subset \mathcal{A}_{n}$. Given an element $a=\left(a_{1}, \ldots, a_{n}\right) \in \mathcal{A}_{n}$ with $a_{j} \in H^{0}\left(C, M^{j}\right)$, called a characteristic, we associate to $a$ a spectral cover of degree $n$

$$
\pi_{a}: X_{a} \longrightarrow C, \quad X_{a}=\operatorname{Zeros}\left(s_{a}\right) \subset|M|,
$$

with

$$
s_{a}=t^{n}+a_{1} t^{n-1}+\cdots+a_{n-1} t+a_{n} \in H^{0}\left(|M|, \pi^{*} M^{n}\right) .
$$

Remark 2.5 Given $a \in \mathcal{A}_{n}$ we observe that the pull-back of the spectral cover $X_{a} \subset$ $|M|$ by the automorphism of $|M|$ given by translation with the section $-\frac{a_{1}}{n}$, that is, $(x, y) \mapsto\left(x, y-\frac{1}{n} a_{1}(x)\right)$, equals the spectral cover $X_{a^{\prime}}$ for some $a^{\prime} \in \mathcal{A}_{n}^{0}$; equivalently do the change of variables $t \mapsto t-\frac{a_{1}}{n}$. Hence $X_{a} \cong X_{a^{\prime}}$. It therefore suffices to restrict our study to spectral covers $X_{a}$ for $a \in \mathcal{A}_{n}^{0}$. 


\subsection{Non-reduced curves}

Let $X$ be an irreducible curve contained in a smooth surface and let $X^{\text {red }}$ denote its underlying reduced curve. Then there exists a global section $s$ of a line bundle such that $X^{\text {red }}=\operatorname{Zeros}(s)$ and an integer $k$ such that $X=\operatorname{Zeros}\left(s^{k}\right)$. We introduce the subschemes $X_{i}=\operatorname{Zeros}\left(s^{i}\right)$ for $i=1, \ldots, k$, so that we have a filtration of $X$ by closed subschemes

$$
X^{\text {red }}=X_{1} \subset X_{2} \subset \cdots \subset X_{k}=X .
$$

In that case we say that $X$ has a nilpotent structure of order $k$. For any integer $i$ we denote by $\mathcal{O}_{X_{i}}$ the structure sheaf of the subscheme $X_{i} \subset X$. Note that $\mathcal{O}_{X_{i}}$ is naturally a $\mathcal{O}_{X}$-module.

We need to recall a result on the local structure of coherent sheaves on non-reduced curves.

Theorem 2.6 (Drézet [8, Théorème 3.4.1]) Let $X$ be a curve with nilpotent structure of order $k$ and let $\mathcal{E}$ be a coherent sheaf over $X$. Then there exists an open subset $V \subset X$ depending on $\mathcal{E}$ and integers $m_{i}$ such that

$$
\mathcal{E}_{\mid V} \stackrel{\sim}{\longrightarrow} \bigoplus_{i=1}^{k} \mathcal{O}_{X_{i}}^{\oplus m_{i}} \mid V .
$$

The sheaf on the right is called a quasi-free sheaf.

\section{The norm map}

In this section we recall the definition of the norm map and prove some of its properties. The standard references are Grothendieck [11, Section 6.5] and [12, Section 21.5].

\subsection{Definition}

Let $C$ be a smooth projective curve and let

$$
\pi: X \longrightarrow C
$$

be any finite degree $n$ covering of $C$. The $\mathcal{O}_{C}$-algebra $\pi_{*} \mathcal{O}_{C}$ will be denoted $\mathcal{B}$ and the group of invertible elements in $\mathcal{B}$ by $\mathcal{B}^{*}$. Note that $\mathcal{B}$ is a locally free sheaf of rank $n$. Let $U \subset C$ be an open subset and let $s \in \Gamma(U, \mathcal{B})=\Gamma\left(\pi^{-1}(U), \mathcal{O}_{X}\right)$ be a local section. One defines (see [11, Section 6.5.1])

$$
N_{X / C}(s):=\operatorname{det}\left(\mu_{s}\right) \in \Gamma\left(U, \mathcal{O}_{C}\right)
$$


where $\mu_{s}: \mathcal{B}_{\mid U} \rightarrow \mathcal{B}_{\mid U}$ is the multiplication with the section $s$. Moreover $s$ is invertible in $\Gamma(U, \mathcal{B})$ if and only if $N_{X / C}(s)$ is invertible in $\Gamma\left(U, \mathcal{O}_{X}\right)$. We have the following obvious relations

$$
N_{X / C}\left(s \cdot s^{\prime}\right)=N_{X / C}(s) \cdot N_{X / C}\left(s^{\prime}\right), \quad N_{X / C}(\lambda s)=\lambda^{n} N_{X / C}(s)
$$

for any local sections $s$ and $s^{\prime}$ of $\mathcal{B}$ and any local section $\lambda$ of $\mathcal{O}_{C}$.

Let $\mathcal{L}$ be an invertible $\mathcal{B}$-module. We can choose a covering $\left\{U_{\lambda}\right\}_{\lambda \in \Lambda}$ of $C$ by open subsets and trivializations $\eta_{\lambda}: \mathcal{L}_{\mid U_{\lambda}} \stackrel{\sim}{\longrightarrow} \mathcal{B}_{\mid U_{\lambda}}$. Then $\left(\omega_{\lambda, \mu}\right)_{\lambda, \mu \in \Lambda}$ with

$$
\omega_{\lambda, \mu}=\eta_{\lambda} \circ \eta_{\mu \mid U_{\lambda} \cap U_{\mu}}^{-1} \in \Gamma\left(U_{\lambda} \cap U_{\mu}, \mathcal{B}\right)
$$

is a 1 -cocycle with values in $\mathcal{B}^{*}$ and $\left(N_{X / C}\left(\omega_{\lambda, \mu}\right)\right)_{\lambda, \mu \in \Lambda}$ is a 1 -cocycle with values in $\mathcal{O}_{C}^{*}$, the sheaf of invertible elements of $\mathcal{O}_{C}$. This 1 -cocycle determines an invertible sheaf over $C$, which we denote by $\operatorname{Nm}_{X / C}(\mathcal{L})$. The following properties easily follow from (7)

$$
\begin{aligned}
\operatorname{Nm}_{X / C}\left(\mathcal{L} \otimes \mathcal{L}^{\prime}\right) & =\operatorname{Nm}_{X / C}(\mathcal{L}) \otimes \operatorname{Nm}_{X / C}\left(\mathcal{L}^{\prime}\right), \\
\operatorname{Nm}_{X / C}\left(\pi^{*} \mathcal{M}\right) & =\mathcal{M}^{\otimes n},
\end{aligned}
$$

for any two invertible sheaves $\mathcal{L}$ and $\mathcal{L}^{\prime}$ over $X$ and for any invertible sheaf $\mathcal{M}$ over $C$. We therefore obtain a group homomorphism between the Picard groups of the curves $X$ and $C$ called the norm map

$$
\operatorname{Nm}_{X / C}: \operatorname{Pic}(X) \longrightarrow \operatorname{Pic}(C), \quad \mathcal{L} \mapsto \operatorname{Nm}_{X / C}(\mathcal{L}) .
$$

\subsection{Properties}

In the case $X$ is smooth, the norm map $\mathrm{Nm}_{X / C}$ has a more explicit description in terms of divisors associated to line bundles.

Proposition 3.1 (Grothendieck [12, Section 21.5]) Assume that $X$ is a smooth curve. The norm map, as defined above, coincides with the map

$$
\mathcal{L}=\mathcal{O}_{X}\left(\sum_{i \in I} n_{i} p_{i}\right) \mapsto \operatorname{Nm}_{X / C}(\mathcal{L})=\mathcal{O}_{C}\left(\sum_{i \in I} n_{i} \pi\left(p_{i}\right)\right),
$$

where $n_{i} \in \mathbb{Z}$ and $p_{i} \in X$. Note that this map is well-defined, that is, $\operatorname{Nm}_{X / C}(\mathcal{L})$ only depends on the linear equivalence class of the divisor $\sum_{i \in I} n_{i} p_{i}$.

From now on the curve $X$ is again an arbitrary cover of $C$. 
Lemma 3.2 Let $0 \rightarrow \mathcal{E} \rightarrow \mathcal{F} \rightarrow \mathcal{T} \rightarrow 0$ be an exact sequence of $\mathcal{O}_{X}$-modules. We assume that $\mathcal{E}$ and $\mathcal{F}$ are torsion-free and that $\mathcal{T}$ is a torsion sheaf. Let $\varphi_{\bullet}$ be a local morphism over $\pi^{-1}(U)$ for some open subset $U \subset C$ between exact sequences

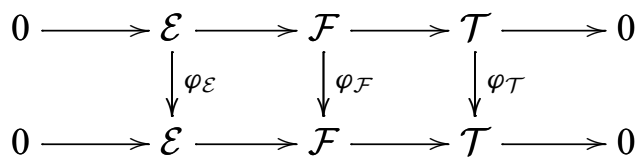

We consider the $\mathcal{O}_{C}$-linear maps induced by $\varphi_{\mathcal{E}}$ and $\varphi_{\mathcal{F}}$ in the direct image sheaves $\pi_{*} \mathcal{E}$ and $\pi_{*} \mathcal{F}$. Then we have the equality

$$
\operatorname{det}\left(\varphi_{\mathcal{E}}\right)=\operatorname{det}\left(\varphi_{\mathcal{F}}\right) \in \Gamma\left(U, \mathcal{O}_{U}\right) .
$$

Proof It is enough to show that the two local sections $\operatorname{det}\left(\varphi_{\mathcal{E}}\right)$ and $\operatorname{det}\left(\varphi_{\mathcal{F}}\right)$ coincide in the local rings $\mathcal{O}_{C, p}$ for every point $p \in U$. We put $A=\mathcal{O}_{C, p}$ and $K=\operatorname{Fr}(A)$ and denote by $E, F$ and $T$ the corresponding $A$-modules of sheaves $\mathcal{E}, \mathcal{F}$ and $\mathcal{T}$. Then $E$ and $F$ are free $A$-modules, hence we have injections $E \hookrightarrow E \otimes_{A} K$ and $F \hookrightarrow F \otimes_{A} K$. Since $T$ is a torsion module, we have $T \otimes_{A} K=0$. Then after localizing (9) at $p \in C$ and taking tensor product with $K$, we obtain the commutative diagram

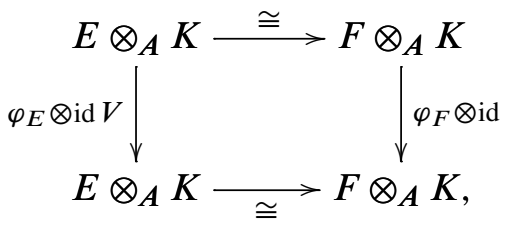

where the horizontal maps are isomorphisms. So $\varphi_{E} \otimes$ id and $\varphi_{F} \otimes$ id are conjugate, hence $\operatorname{det}\left(\varphi_{E} \otimes \mathrm{id}\right)=\operatorname{det}\left(\varphi_{F} \otimes \mathrm{id}\right) \in K$. On the other hand $\operatorname{det}\left(\varphi_{E} \otimes \mathrm{id}\right)$ and $\operatorname{det}\left(\varphi_{F} \otimes\right.$ id $)$ are elements in $A \subset K$, hence we obtain the desired equality.

In the sequel we will use the following properties of the norm map:

Corollary 3.3 Let $\mathcal{E}$ and $\mathcal{F}$ be two torsion-free $\mathcal{O}_{X}$-modules such that

$$
0 \longrightarrow \mathcal{E} \longrightarrow \mathcal{F} \longrightarrow \mathcal{T} \longrightarrow 0
$$

where $\mathcal{T}$ is a torsion $\mathcal{O}_{X}$-module. Let $s \in \Gamma(U, \mathcal{B})=\Gamma\left(\pi^{-1}(U), \mathcal{O}_{X}\right)$ be a local section of $\mathcal{B}$ over the open subset $U \subset C$. We consider the maps induced by the multiplication with the section $s$ in the direct image sheaves $\pi_{*} \mathcal{E}$ and $\pi_{*} \mathcal{F}$, which we denote by $\mu_{s}^{\mathcal{E}} \in \operatorname{Hom}_{\mathcal{O}_{C}(U)}\left(\pi_{*} \mathcal{E}(U), \pi_{*} \mathcal{E}(U)\right)$ and $\mu_{s}^{\mathcal{F}} \in \operatorname{Hom}_{\mathcal{O}_{C}(U)}\left(\pi_{*} \mathcal{F}(U), \pi_{*} \mathcal{F}(U)\right)$. Then we have the equality

$$
\operatorname{det}\left(\mu_{s}^{\mathcal{E}}\right)=\operatorname{det}\left(\mu_{s}^{\mathcal{F}}\right) \in \Gamma\left(U, \mathcal{O}_{C}\right) .
$$


Lemma 3.4 Let $p: \tilde{X} \rightarrow X$ be a covering such that the cokernel of the canonical inclusion $\mathcal{O}_{X} \hookrightarrow p_{*} \mathcal{O}_{\tilde{X}}$ is a torsion $\mathcal{O}_{X}$-module. Then, for any invertible sheaf $\mathcal{L}$ over $X$ we have

$$
\operatorname{Nm}_{\tilde{X} / C}\left(p^{*} \mathcal{L}\right)=\operatorname{Nm}_{X / C}(\mathcal{L})
$$

Proof We consider the exact sequence

$$
0 \longrightarrow \mathcal{O}_{X} \longrightarrow p_{*} \mathcal{O}_{\tilde{X}} \longrightarrow \mathcal{T} \longrightarrow 0,
$$

where $\mathcal{T}$ is a torsion $\mathcal{O}_{X}$-module. Note that the direct image $p_{*} \mathcal{O}_{\tilde{X}}$ is torsion-free. We denote the $\mathcal{O}_{C}$-algebra $\pi_{*} p_{*} \mathcal{O}_{\tilde{X}}$ by $\tilde{\mathcal{B}}$. Note that $\tilde{\mathcal{B}}$ is a $\mathcal{B}$-module. Let $\mathcal{L}$ be an invertible $\mathcal{O}_{X}$-module, $\eta_{\lambda}: \mathcal{L}_{\mid U_{\lambda}} \stackrel{\sim}{\longrightarrow} \mathcal{B}_{\mid U_{\lambda}}$ be a set of trivializations of $\mathcal{L}$ as $\mathcal{B}$-module, and $\left(\omega_{\lambda, \mu}\right)_{\lambda, \mu \in \Lambda}$ be the corresponding 1 -cocycle with values in $\mathcal{B}^{*}$. Then the pull-back $p^{*} \mathcal{L}$ corresponds to a 1 -cocycle $\left(p^{*} \omega_{\lambda, \mu}\right)_{\lambda, \mu \in \Lambda}$ with values in $\widetilde{\mathcal{B}}^{*}$ obtained from $\left(\omega_{\lambda, \mu}\right)_{\lambda, \mu \in \Lambda}$ under the canonical inclusion $\mathcal{B} \hookrightarrow \widetilde{\mathcal{B}}$. We now apply Corollary 3.3 to the exact sequence (10) and conclude that $N_{\tilde{X} / C}\left(p^{*} \omega_{\lambda, \mu}\right)=$ $N_{X / C}\left(\omega_{\lambda, \mu}\right) \in \Gamma\left(U_{\lambda} \cap U_{\mu}, \mathcal{O}_{C}\right)$. This proves the lemma.

Lemma 3.5 Let $X=\bigcup_{i=1}^{r} X_{i}$ be the decomposition of $X$ into irreducible components $X_{i}$. For an invertible sheaf $\mathcal{L}$, we denote by $\mathcal{L}_{i}=\mathcal{L} \otimes_{\mathcal{O}_{X}} \mathcal{O}_{X_{i}}$ its restriction to $X_{i}$. Then, we have the equality

$$
\mathrm{Nm}_{X / C}(\mathcal{L})=\bigotimes_{i=1}^{r} \mathrm{Nm}_{X_{i} / C}\left(\mathcal{L}_{i}\right)
$$

Proof We apply the previous lemma to the covering $p: \tilde{X}=\bigsqcup_{i=1}^{r} X_{i} \rightarrow X$ given by the disjoint union of the curves $X_{i}$.

Lemma 3.6 Let $X$ be an irreducible curve and let $j: X^{\text {red }} \hookrightarrow X$ be its underlying reduced curve. Let $m$ be the multiplicity of $X^{\text {red }}$ in $X$. Then, for any invertible sheaf $\mathcal{L}$ over $X$ we have

$$
\operatorname{Nm}_{X / C}(\mathcal{L})=\mathrm{Nm}_{X^{\mathrm{red}} / C}\left(j^{*} \mathcal{L}\right)^{\otimes m} .
$$

Proof The $\mathcal{O}_{C}$-algebra $\mathcal{B}=\pi_{*} \mathcal{O}_{X}$ comes equipped with a nilpotent ideal sheaf $\mathcal{J} \subset \mathcal{B}$ such that $\mathcal{B}_{\text {red }}=\mathcal{B} / \mathcal{J}=\pi_{*} \mathcal{O}_{X^{\text {red }}}$. We choose a covering $\left\{U_{\lambda}\right\}_{\lambda \in \Lambda}$ of $C$ by open subsets which trivialize the invertible sheaf $\mathcal{L}$, that is, there exists isomorphisms $\eta_{\lambda}: \mathcal{L}_{\mid U_{\lambda}} \stackrel{\sim}{\longrightarrow} \mathcal{B}_{\mid U_{\lambda}}$ and such that $\mathcal{J}_{\mid U_{\lambda}}$ is generated by an element $t \in \mathcal{B}_{\mid U_{\lambda}}$. Then multiplication with the invertible element $\omega_{\lambda, \mu}=\eta_{\lambda} \circ \eta_{\mu \mid U_{\lambda} \cap U_{\mu}}^{-1}$ preserves the filtration $t^{m-1} \mathcal{B}_{\mid U_{\lambda}} \subset \cdots \subset t \mathcal{B}_{\mid U_{\lambda}} \subset \mathcal{B}_{\mid U_{\lambda}}$ and acts on the quotients as multiplication with $\omega_{\lambda, \mu}^{\text {red }} \in \mathcal{B}_{\mid U_{\lambda} \cap U_{\mu}}^{\text {red }}$. It follows that $N_{X / C}\left(\omega_{\lambda, \mu}\right)=N_{X^{\text {red }} / C}\left(\omega_{\lambda, \mu}^{\text {red }}\right)^{m}$, which proves the lemma. 


\subsection{The Prym variety $\operatorname{Prym}(X / C)$}

Given a spectral cover $\pi: X \rightarrow C$ we denote by $\operatorname{Pic}^{0}(X)$ the connected component of the identity element of the Picard group of $X$ (see, for example, Kleiman [22]). We then define the Prym variety $\operatorname{Prym}(X / C)$ to be the kernel of the Norm map $\mathrm{Nm}_{X / C}$

$$
\operatorname{Prym}(X / C):=\operatorname{ker}\left(\operatorname{Nm}_{X / C}: \operatorname{Pic}^{0}(X) \longrightarrow \operatorname{Pic}^{0}(C)\right) .
$$

We recall that $n$ denotes the degree of the cover $\pi: X \rightarrow C$. We choose an ample line bundle $\mathcal{O}_{C}(1)$ over $C$ and denote by $\mathcal{O}_{X}(1)=\pi^{*} \mathcal{O}_{C}(1)$ its pull-back to $X$ and by $\delta=\operatorname{deg} \mathcal{O}_{C}(1)$.

Definition 3.7 Let $\mathcal{E}$ be a coherent $\mathcal{O}_{X}$-module. The rank and degree of $\mathcal{E}$ with respect to the polarization $\mathcal{O}_{X}(1)$ are the rational numbers $\operatorname{rk}(\mathcal{E})$ and $\operatorname{deg}(\mathcal{E})$ determined by the Hilbert polynomial

$$
\chi\left(X, \mathcal{E} \otimes \mathcal{O}_{X}(l)\right)=n \delta l \operatorname{rk}(\mathcal{E})+\operatorname{deg}(\mathcal{E})+\operatorname{rk}(\mathcal{E}) \chi\left(\mathcal{O}_{X}\right) .
$$

The slope of $\mathcal{E}$ is defined by $\mu(\mathcal{E})=\frac{\operatorname{deg}(\mathcal{E})}{\operatorname{rk}(\mathcal{E})}$. The sheaf $\mathcal{E}$ is stable (resp. semi-stable) if $\mathcal{E}$ is torsion-free and for any proper subsheaf $\mathcal{E}^{\prime} \subset \mathcal{E}$ we have the equality $\mu\left(\mathcal{E}^{\prime}\right)<\mu(\mathcal{E})$ (resp. $\leq$ ).

Remark 3.8 The definitions of rank and degree of a coherent sheaf $\mathcal{E}$ over $X$ above coincide with the classical ones when the curve $X$ is integral. The (semi-)stability condition above coincides with the (semi-)stability condition introduced by Simpson [31].

Remark 3.9 Using the equality $\chi\left(X, \mathcal{E} \otimes \mathcal{O}_{X}(l)\right)=\chi\left(C, \pi_{*} \mathcal{E} \otimes \mathcal{O}_{C}(l)\right)$ we obtain the following formulae

$$
\text { and } \quad \begin{aligned}
\operatorname{nrk}(\mathcal{E}) & =\operatorname{rk}\left(\pi_{*} \mathcal{E}\right) \\
\operatorname{deg}(\mathcal{E})+\operatorname{rk}(\mathcal{E}) \chi\left(\mathcal{O}_{X}\right) & =\operatorname{deg}\left(\pi_{*} \mathcal{E}\right)+\operatorname{rk}\left(\pi_{*} \mathcal{E}\right) \chi\left(\mathcal{O}_{C}\right) .
\end{aligned}
$$

Proposition 3.10 Let $\mathcal{E}$ be a torsion-free $\mathcal{O}_{X}$-module of integral rank $r=\operatorname{rk}(\mathcal{E})$ and let $\mathcal{L}$ be an invertible $\mathcal{O}_{X}$-module. Then we have the relation

$$
\operatorname{det}\left(\pi_{*}(\mathcal{E} \otimes \mathcal{L})\right)=\operatorname{det}\left(\pi_{*} \mathcal{E}\right) \otimes \mathrm{Nm}_{X / C}(\mathcal{L})^{\otimes r} .
$$

Proof We shall use the notation of Section 3.1. Since $\mathcal{E}$ is torsion-free, the direct image $\pi_{*} \mathcal{E}$ is a locally free $\mathcal{O}_{C}$-module. We choose a covering $\left\{U_{\lambda}\right\}_{\lambda \in \Lambda}$ of $C$ for which both $\mathcal{L}$ and $\pi_{*} \mathcal{E}$ are trivialized, that is, such that there exists local isomorphisms

$$
\alpha_{\lambda}: \pi_{*} \mathcal{E}_{\mid U_{\lambda}} \stackrel{\sim}{\longrightarrow} \mathcal{O}_{U_{\lambda}}^{\oplus r n} \quad \text { and } \quad \tau_{\lambda}: \mathcal{L}_{\mid U_{\lambda}} \stackrel{\sim}{\longrightarrow} \mathcal{B}_{U_{\lambda}} .
$$


Since $\mathcal{L}$ is trivial on $U_{\lambda}$ we have an isomorphism

$$
\operatorname{id}_{\mathcal{E}} \otimes \tau_{\lambda}: \mathcal{E} \otimes \mathcal{L}_{\mid U_{\lambda}} \longrightarrow \mathcal{E} \otimes \mathcal{B}_{\mid U_{\lambda}}
$$

which we can consider as an isomorphism between $\mathcal{O}_{C}$-modules

$$
\operatorname{id}_{\mathcal{E}} \otimes \tau_{\lambda}: \pi_{*}(\mathcal{E} \otimes \mathcal{L})_{\mid U_{\lambda}} \longrightarrow \pi_{*} \mathcal{E}_{\mid U_{\lambda}} .
$$

We compose with $\alpha_{\lambda}$ to obtain a trivialization of $\pi_{*}(\mathcal{E} \otimes \mathcal{L})_{\mid U_{\lambda}}$

$$
\beta_{\lambda}: \pi_{*}(\mathcal{E} \otimes \mathcal{L})_{\mid U_{\lambda}} \stackrel{\mathrm{id} \mathcal{E}_{\mathcal{E}} \otimes \tau_{\lambda}}{\longrightarrow} \pi_{*} \mathcal{E}_{\mid U_{\lambda}} \stackrel{\alpha_{\lambda}}{\longrightarrow} \mathcal{O}_{U_{\lambda}}^{\oplus r n}
$$

Given $\lambda, \mu \in \Lambda$ we can now write the transition functions $f_{\lambda, \mu}=\beta_{\lambda} \circ \beta_{\mu}^{-1}$ of the vector bundle $\pi_{*}(\mathcal{E} \otimes \mathcal{L})$ as

$$
f_{\lambda, \mu}: \mathcal{O}_{U_{\lambda}}^{\oplus r n} \stackrel{\alpha_{\mu}^{-1}}{\longrightarrow}\left(\pi_{*} \mathcal{E}\right)_{\mid U_{\lambda, \mu}} \stackrel{\mathrm{id}_{\mathcal{E}} \otimes \omega_{\lambda, \mu}}{\longrightarrow}\left(\pi_{*} \mathcal{E}\right)_{\mid U_{\lambda, \mu}} \stackrel{\alpha_{\lambda}}{\longrightarrow} \mathcal{O}_{U_{\lambda}}^{\oplus r n},
$$

where we denote by $\omega_{\lambda, \mu}=\tau_{\lambda} \circ \tau_{\mu}^{-1}$ the $\mathcal{B}^{*}$-valued transition functions of the line bundle $\mathcal{L}$. We deduce from this expression the relation

$$
\operatorname{det}\left(f_{\lambda, \mu}\right)=\operatorname{det}\left(g_{\lambda, \mu}\right) \cdot \operatorname{det}\left(\operatorname{id}_{\mathcal{E}} \otimes \omega_{\lambda, \mu}\right),
$$

where $g_{\lambda, \mu}=\alpha_{\lambda} \circ \alpha_{\mu}^{-1}$ denotes the transition functions of the vector bundle $\pi_{*} \mathcal{E}$. Hence the proposition follows if we show the relation $\operatorname{det}\left(\mathrm{id}_{\mathcal{E}} \otimes \omega_{\lambda, \mu}\right)=\operatorname{det}\left(\omega_{\lambda, \mu}\right)^{r}$, which is proved in the next Lemma.

Lemma 3.11 Let $\mathcal{E}$ be a torsion-free $\mathcal{O}_{X}$-module and let

$$
s \in \Gamma(U, \mathcal{B})=\Gamma\left(\pi^{-1}(U), \mathcal{O}_{X}\right)
$$

be a local section of $\mathcal{B}$ over the open subset $U \subset C$. We denote by

$$
\mu_{s}^{\mathcal{E}} \in \operatorname{Hom}_{\mathcal{O}_{C}(U)}\left(\pi_{*} \mathcal{E}(U), \pi_{*} \mathcal{E}(U)\right)
$$

the map induced by multiplication with the section $s$. Then we have an equality

$$
\operatorname{det}\left(\mu_{s}^{\mathcal{E}}\right)=\operatorname{det}\left(\mu_{s}\right)^{r} \in \Gamma\left(U, \mathcal{O}_{C}\right) .
$$

Proof By Theorem 2.6 there exists an open subset $j: V \hookrightarrow X$ such that $j^{*} \mathcal{E}$ is isomorphic to $j^{*} \mathcal{Q}$ where $\mathcal{Q}$ is a quasi-free sheaf of the form $\bigoplus_{i=1}^{k} \mathcal{O}_{X_{i}}^{\oplus m_{i}}$. We then apply Corollary 3.3 to the two exact sequences

$$
0 \longrightarrow \mathcal{E} \longrightarrow j_{*} j^{*} \mathcal{E} \longrightarrow \mathcal{T}_{1} \longrightarrow 0 \quad \text { and } \quad 0 \longrightarrow \mathcal{Q} \longrightarrow j_{*} j^{*} \mathcal{Q} \longrightarrow \mathcal{T}_{2} \longrightarrow 0
$$


where $\mathcal{T}_{i}$ are torsion sheaves. This leads to the equality $\operatorname{det}\left(\mu_{s}^{\mathcal{E}}\right)=\operatorname{det}\left(\mu_{s}^{\mathcal{Q}}\right)$. It therefore suffices to compute $\operatorname{det}\left(\mu_{s}^{\mathcal{Q}}\right)$ in terms of $\operatorname{det}\left(\mu_{s}\right)$. We put $n=k \cdot l$ with $l=\operatorname{deg}\left(X^{\text {red }} / C\right)$. Then we have

$$
r=\operatorname{rk}(\mathcal{E})=\operatorname{rk}(\mathcal{Q})=\frac{1}{n} \sum_{i=1}^{k} m_{i} \operatorname{rk}\left(\pi_{*} \mathcal{O}_{X_{i}}\right)=\frac{1}{n} \sum_{i=1}^{k} m_{i} i l=\frac{1}{k} \sum_{i=1}^{k} m_{i} i .
$$

Let $A=\mathcal{O}_{C, p}$ denote the local ring at the point $p \in C$ and let $B$ denote the localization of $\pi_{*} \mathcal{O}_{X}$ at the point $p \in C$. Thus $B$ is a projective $A$-module of rank $n$ equipped with a filtration

$$
t^{k-1} B \subset \cdots \subset t B \subset B, \quad t \in B \text { with } t^{k}=0 .
$$

We put $B_{1}=B / t B$, the localization of $\pi_{*} \mathcal{O}_{X^{\text {red }}}$ at the point $p \in C$. Since $B$ is projective we can choose a splitting

$$
B=B_{1} \oplus t B_{1} \oplus \cdots \oplus t^{k-1} B_{1} .
$$

Using this decomposition we can write a section $s \in B$ as $s=s_{0}+t s_{1}+\cdots+t^{k-1} s_{k-1}$ with $s_{j} \in B_{1}$. Moreover, the localization of $\pi_{*} \mathcal{O}_{X_{i}}$ at the point $p \in C$ is given by $B_{i}:=B_{1} \oplus t B_{1} \oplus \cdots \oplus t^{i-1} B_{1}$ and the matrix of the multiplication with $s$ in $B_{i}$ is with respect to this decomposition lower block-triangular and has determinant $\operatorname{det}\left(\mu_{s}^{B_{i}}\right)=\operatorname{det}\left(\mu_{s_{0}}^{B_{1}}\right)^{i}$. Therefore

$$
\operatorname{det}\left(\mu_{s}^{\mathcal{Q}}\right)=\prod_{i=1}^{k} \operatorname{det}\left(\mu_{s}^{B_{i}}\right)^{m_{i}}=\operatorname{det}\left(\mu_{s_{0}}^{B_{1}}\right)^{\sum_{i=1}^{k} i m_{i}} .
$$

On the other hand $\operatorname{det}\left(\mu_{s}\right)=\operatorname{det}\left(\mu_{s}^{\mathcal{O}_{X}}\right)=\operatorname{det}\left(\mu_{s}^{B_{k}}\right)=\operatorname{det}\left(\mu_{s_{0}}^{B_{1}}\right)^{k}$, which leads to the desired equality.

Taking the trivial sheaf $\mathcal{E}=\mathcal{O}_{X}$ in Proposition 3.10 we obtain the following description of the norm map:

Corollary 3.12 For any invertible $\mathcal{O}_{X}$-module, we have

$$
\mathrm{Nm}_{X / C}(\mathcal{L})=\operatorname{det}\left(\pi_{*} \mathcal{L}\right) \otimes \operatorname{det}\left(\pi_{*} \mathcal{O}_{X}\right)^{-1}
$$

\section{The group of connected components of $\operatorname{Prym}(X / C)$}

In this section we give the proof of Theorem 1.1. 


\subsection{Proof of Theorem $1.1(1)$}

Given a spectral cover $X$ we will associate a covering

$$
p: \tilde{X} \longrightarrow X
$$

as follows: let $X=\bigcup_{i=1}^{r} X_{i}$ be its decomposition into irreducible components $X_{i}$, let $X_{i}^{\text {red }}$ be the underlying reduced curve of $X_{i}$, let $m_{i}$ be the multiplicity of $X_{i}^{\text {red }}$ in $X_{i}$ and let $\tilde{X}_{i}^{\text {red }}$ be the normalization of $X_{i}^{\text {red }}$. Since $X_{i}^{\text {red }}$ is embedded in the smooth surface $|M|$, there exists a sequence of blowing-ups bl: $\widetilde{|M|} \rightarrow|M|$ of the surface $|M|$ at reduced points (depending on the curve $X_{i}^{\text {red }}$ ) such that the proper transform of $X_{i}^{\text {red }}$ equals its normalization $\tilde{X}_{i}^{\text {red }}$. We then define $\tilde{X}_{i} \subset \widetilde{|M|}$ to be the proper transform of the non-reduced curve $X_{i} \subset|M|$, and take

$$
\tilde{X}=\bigsqcup_{i=1}^{r} \tilde{X}_{i}
$$

to be the disjoint union of the curves $\tilde{X}_{i}$ together with the natural map $p$ onto $X$. Note that the multiplicity of $\tilde{X}_{i}^{\text {red }}$ in $\tilde{X}_{i}$ also equals $m_{i}$.

Lemma 4.1 The covering $p: \tilde{X} \rightarrow X$ constructed above has the following properties:

(1) the cokernel of the canonical inclusion $\mathcal{O}_{X} \hookrightarrow p_{*} \mathcal{O}_{\tilde{X}}$ is a torsion $\mathcal{O}_{X}$-module,

(2) the underlying reduced curve $\tilde{X}^{\text {red }}$ of $\tilde{X}$ is smooth.

(3) the map induced by pull-back under $p$

$$
\operatorname{Pic}^{0}(X) \stackrel{p^{*}}{\longrightarrow} \operatorname{Pic}^{0}(\tilde{X})
$$

is surjective and has connected kernel.

(4) we have an equality

$$
\pi_{0}(\operatorname{Prym}(X / C))=\pi_{0}(\operatorname{Prym}(\tilde{X} / C)) .
$$

\section{Proof}

(1) This is clear since $p: \tilde{X} \rightarrow X$ is an isomorphism outside a finite set of points.

(2) We clearly have $\tilde{X}^{\text {red }}=\bigsqcup_{i=1}^{r} \tilde{X}_{i}^{\text {red }}$ and the curves $\tilde{X}_{i}^{\text {red }}$ are smooth by construction. 
(3) We consider the two exact sequences obtained by restricting invertible sheaves to the underlying reduced curve

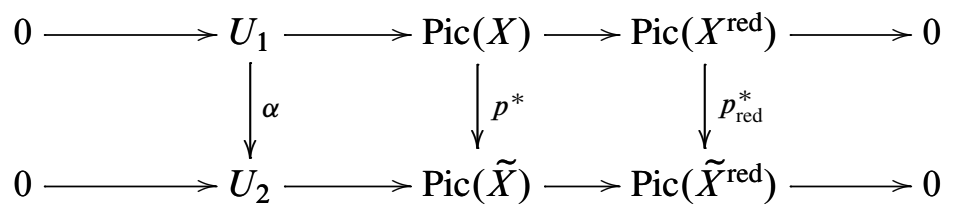

which are surjective with unipotent kernels $U_{1}$ and $U_{2}$ by Liu [23, Lemma 7.5.11]. Then by the snake lemma the $\operatorname{kernel} \operatorname{ker}\left(p^{*}\right)$ fits into the exact sequence

$$
0 \longrightarrow \operatorname{ker}(\alpha) \longrightarrow \operatorname{ker}\left(p^{*}\right) \longrightarrow \operatorname{ker}\left(p_{\text {red }}^{*}\right) \longrightarrow \operatorname{coker}(\alpha) \longrightarrow 0
$$

Note that $\operatorname{ker}(\alpha)$ and $\operatorname{coker}(\alpha)$ are unipotent groups. We shall denote by $V$ the kernel of the last map. By [23, Lemma 7.5.13] the $\operatorname{kernel} \operatorname{ker}\left(p_{\text {red }}^{*}\right)$ is an extension of a toric group by an unipotent group. The same holds for $V$, since there are no non-zero maps from a toric group to an unipotent group. Hence $V$ and $\operatorname{ker}(\alpha)$ are connected, so $\operatorname{ker}\left(p^{*}\right)$ is connected. Hence $\operatorname{ker}\left(p^{*}\right)$ is contained in the connected component $\operatorname{Pic}^{0}(X)$ and we obtain that $p^{*}: \operatorname{Pic}^{0}(X) \rightarrow \operatorname{Pic}^{0}(\tilde{X})$ is surjective.

(4) Because of Lemma 3.4 we have an exact sequence

$$
0 \longrightarrow \operatorname{ker}\left(p^{*}\right) \longrightarrow \operatorname{Prym}(X / C) \stackrel{p^{*}}{\longrightarrow} \operatorname{Prym}(\tilde{X} / C) \longrightarrow 0 .
$$

The equality between the groups of connected components now follows since $\operatorname{ker}\left(p^{*}\right)$ is connected.

The previous lemma implies that it is enough to show the equality $\pi_{0}(\operatorname{Prym}(\tilde{X} / C))=$ $\widehat{K}$. By Lemma 3.5 and Lemma 3.6 the Norm map $\mathrm{Nm}_{\tilde{X} / C}$ factorizes as follows:

$\operatorname{Nm}_{\tilde{X} / C}: \operatorname{Pic}^{0}(\tilde{X}) \stackrel{j^{*}}{\longrightarrow} \operatorname{Pic}^{0}\left(\tilde{X}^{\mathrm{red}}\right)=\prod_{i=1}^{r} \operatorname{Pic}^{0}\left(\tilde{X}_{i}^{\mathrm{red}}\right) \stackrel{\prod\left[m_{i}\right]}{\longrightarrow} \prod_{i=1}^{r} \operatorname{Pic}^{0}\left(\tilde{X}_{i}^{\mathrm{red}}\right) \stackrel{\prod \mathrm{Nm}}{\longrightarrow} \operatorname{Pic}^{0}(C)$

Moreover $j^{*}$ is surjective and $\operatorname{ker}\left(j^{*}\right)$ is connected (see, for example, Liu [23, Lemma 7.5.11]). It suffices therefore to compute $\pi_{0}(\operatorname{ker}(h))$, where $h: \operatorname{Pic}^{0}\left(\tilde{X}^{\text {red }}\right) \rightarrow$ $\operatorname{Pic}^{0}(C)$ denotes the composite of the last two maps. We also consider the composite homomorphism

$$
f: \operatorname{Pic}^{0}(C) \stackrel{\Delta}{\longrightarrow} \operatorname{Pic}^{0}(C)^{r} \stackrel{\prod\left[m_{i}\right]}{\longrightarrow} \operatorname{Pic}^{0}(C)^{r} \stackrel{\prod \tilde{\pi}_{i}^{*}}{\longrightarrow} \prod_{i=1}^{r} \operatorname{Pic}^{0}\left(\tilde{X}_{i}^{\text {red }}\right)=\operatorname{Pic}^{0}\left(\tilde{X}^{\mathrm{red}}\right)
$$


where $\Delta(L)=(L, \ldots, L)$ is the diagonal map. We note that the duals $\hat{\widetilde{\pi}}_{i}^{*}$ and $\widehat{\left[m_{i}\right]}$ coincide with $\mathrm{Nm}_{\tilde{X}_{i}^{\text {red }} / C}$ and $\left[m_{i}\right]$ under the identifications

$$
\widehat{\operatorname{Pic}^{0}(C)} \cong \operatorname{Pic}^{0}(C) \quad \text { and } \quad \widehat{\operatorname{Pic}^{0}\left(\widetilde{X}_{i}^{\text {red }}\right)} \cong \operatorname{Pic}^{0}\left(\tilde{X}_{i}^{\text {red }}\right)
$$

given by the principal polarizations on the Jacobians (see Birkenhake and Lange [4, Section 11.4]), and that the dual $\widehat{\Delta}$ of $\Delta$ is the multiplication map on $\operatorname{Pic}^{0}(C)$ (see, for example, [4, Exercise 2.6(12)]). Hence we obtain that $\hat{f}=h$. Thus $\pi_{0}(\operatorname{Prym}(\tilde{X} / C))=$ $\pi_{0}(\operatorname{ker}(\hat{f}))$. Now we apply Lemma 2.1 to $f$ and we obtain the desired result since $\operatorname{ker}(f)=\bigcap_{i=1}^{r}\left(K_{i}\right)_{m_{i}}$.

\subsection{Proof of Theorem $1.1(2)$}

We consider the morphism $f: \operatorname{Pic}^{0}(C) \rightarrow \operatorname{Pic}^{0}\left(\tilde{X}^{\text {red }}\right)$ introduced in the previous section. Moreover the morphism $g: \operatorname{Pic}^{0}\left(\tilde{X}^{\text {red }}\right) \rightarrow \operatorname{Pic}^{0}(C)$ defined by

$$
g\left(\mathcal{L}_{1}, \ldots, \mathcal{L}_{r}\right)=\bigotimes_{i=1}^{r} \mathrm{Nm}_{\tilde{X}_{i}^{\text {red }} / C}\left(\mathcal{L}_{i}\right)
$$

satisfies the relation $g \circ f=[n]$. We are therefore in a position to apply Lemma 2.2 to the morphism $f$. This proves part (2) for the $\operatorname{Prym}$ variety $\operatorname{Prym}(\tilde{X} / C)$. Since by Lemma 4.1 the natural map $p^{*}: \operatorname{Pic}^{0}(X) \rightarrow \operatorname{Pic}^{0}(\tilde{X})$ induces an isomorphism $\pi_{0}(\operatorname{Prym}(X / C))=\pi_{0}(\operatorname{Prym}(\tilde{X} / C))$, we are done.

\subsection{Proof of Theorem $1.1(3)$}

The if part follows immediately from the formula proved in part (1). Suppose now that $K=\operatorname{Pic}^{0}(C)[n]$. With the notation above we have $n=\sum_{i=1}^{r} m_{i} \operatorname{deg}\left(X_{i}^{\text {red }} / C\right)$ and $K=\bigcap_{i=1}^{r}\left(K_{i}\right)_{m_{i}}$, from which we deduce that $r=1$. On the other hand $K=$ $\left(K_{1}\right)_{m_{1}}=\operatorname{Pic}^{0}(C)[n]$ implies that $K_{1}=\operatorname{Pic}^{0}(C)\left[d_{1}\right]$ with $d_{1}=\operatorname{deg}\left(X_{1}^{\text {red }} / C\right)$. But this can only happen if $d_{1}=1$. Hence $m_{1}=n$ and we are done.

\section{Endoscopic subloci of $\mathcal{A}_{n}$}

\subsection{Cyclic Galois covers}

We consider a smooth projective curve $C$ and a line bundle $M \in \operatorname{Pic}(C)$. Let $\Gamma$ be a cyclic subgroup of order $d$ of the group of $n$-torsion line bundles $\operatorname{Pic}^{0}(C)[n]$ and let

$$
\varphi: D \longrightarrow C
$$


be the étale Galois covering of $C$ associated to $\Gamma \subset \operatorname{Pic}^{0}(C)[n]$. By definition $D=$ $\operatorname{Spec}\left(\mathcal{E}_{\Gamma}\right)$ where $\mathcal{E}_{\Gamma}=\bigoplus_{L \in \Gamma} L$ is the direct sum of all line bundles $L$ in $\Gamma$ with the natural $\mathcal{O}_{C}$-algebra structure. Note that the Galois group of the covering $\varphi: D \rightarrow C$ equals $\Gamma \cong \operatorname{Aut}(D / C)$. We introduce the line bundle $N=\varphi^{*} M$. Then the line bundle $N$ has a canonical $\Gamma$-linearization, hence we obtain a canonical action of $\Gamma$ on the total space $|N|$. We notice that the canonical coordinate $t \in H^{0}\left(|N|, \pi^{*} N\right)$ is invariant under this $\Gamma$-action.

We consider a spectral cover of degree $m$ over $D$ with associated line bundle $N$ given by a global section $s \in H^{0}\left(|N|, \pi^{*} N^{m}\right)$. We can apply a Galois automorphism $\sigma \in \Gamma$ to $s$ and denote its image by $s^{\sigma}$. We introduce

$$
\widehat{s}=\prod_{\sigma \in \Gamma} s^{\sigma} \in H^{0}\left(|N|, \pi^{*} N^{n}\right), \quad \text { with } n=d \cdot m .
$$

We observe that $\widehat{s}$ is $\Gamma$-invariant, hence $\widehat{s}$ descends to a section over $|M|$, which we also denote by $\widehat{s}$. Hence we obtain a map

$$
\Phi_{\Gamma}: \mathcal{A}_{m}(D, N) \longrightarrow \mathcal{A}_{n}:=\mathcal{A}_{n}(C, M), \quad b \mapsto \Phi_{\Gamma}(b) .
$$

with $a=\Phi_{\Gamma}(b)$ defined by the relation $\widehat{s}_{b}=s_{a}$, where $s_{b} \in H^{0}\left(|N|, \pi^{*} N^{m}\right)$ is the global section $s_{b}=t^{m}+b_{1} t^{m-1}+b_{2} t^{m-2}+\cdots+b_{m}$ associated to $b=\left(b_{1}, \ldots, b_{m}\right)$ with $b_{j} \in H^{0}\left(D, N^{j}\right)$. Since the zero divisor of the section $\widehat{s}$ has a finite number of irreducible components, we immediately see that the fiber $\Phi_{\Gamma}^{-1}(\widehat{s})$ is finite, hence $\operatorname{dim} \operatorname{im} \Phi_{\Gamma}=\operatorname{dim} \mathcal{A}_{m}(D, N)$. We also introduce the subspace

$$
\mathcal{A}_{m}^{\Gamma}(D, N)=H^{0}(D, N)_{v a r} \oplus \bigoplus_{j=2}^{m} H^{0}\left(D, N^{j}\right) \subset \mathcal{A}_{m}(D, N),
$$

where $H^{0}(D, N)_{\text {var }}$ denotes the $\Gamma$-variant subspace of $H^{0}(D, N)$, that is, the direct sum of the character spaces $H^{0}(D, N)_{\chi}$ for non-trivial characters $\chi$ of the group $\Gamma$.

Lemma 5.1 We have the inclusion

$$
\Phi_{\Gamma}\left(\mathcal{A}_{m}^{\Gamma}(D, N)\right) \subset \mathcal{A}_{n}^{0} .
$$

Proof It suffices to compute the coefficient of $t^{n-1}$ in $\widehat{s}_{b}$, which equals $\sum_{\sigma \in \Gamma} \sigma^{*} b_{1}$. We immediately see that the relation $\sum_{\sigma \in \Gamma} \sigma^{*} b_{1}=0$ is equivalent to $b_{1}^{(0)}=0$, where $b_{1}^{(0)}$ denotes the $\Gamma$-invariant component of $b_{1}$.

We denote the images of $\Phi_{\Gamma}$ by

$$
\mathcal{A}_{\Gamma}^{0} \subset \mathcal{A}_{n}^{0} \quad \text { and } \quad \mathcal{A}_{\Gamma} \subset \mathcal{A}_{n} .
$$


The subvariety $\mathcal{A}_{\Gamma}$ admits the following characterization: for $a \in \mathcal{A}_{n}$ we denote by

$$
Y_{a}=X_{a} \times_{C} D
$$

the fiber product of $X_{a}$ and $D$ over $C$. Then $Y_{a}$ is a spectral cover over $D$ of degree $n$ associated to the line bundle $N$. The following lemma follows immediately from the definition of $\mathcal{A}_{\Gamma}$.

Lemma 5.2 The characteristic a lies in $\mathcal{A}_{\Gamma}$ if ond only if the fiber product $Y_{a}$ decomposes as

$$
Y_{a}=\bigcup_{\sigma \in \Gamma} Z^{\sigma}
$$

where $Z$ is a spectral cover of degree $m=\frac{n}{d}$ over $D$ and $Z^{\sigma}$ is its image under the Galois automorphism $\sigma \in \Gamma$.

We also need to introduce some natural subvarieties of the Hitchin spaces $\mathcal{A}_{n}^{0}$ and $\mathcal{A}_{n}$, which will be used in the proof of Theorem 1.2.

For any divisor $l \neq 1$ of $n$, with $n=k \cdot l$, we consider the natural $k$ th power map

$$
\Phi_{k}: \mathcal{A}_{l} \longrightarrow \mathcal{A}_{n}
$$

where $\Phi_{k}(b)=a$ is defined by the relation

$$
s_{a}=\left(t^{l}+b_{1} t^{l-1}+\cdots+b_{l}\right)^{k} \in H^{0}\left(|M|, \pi^{*} M^{n}\right), \quad \text { for } b=\left(b_{1}, \ldots, b_{l}\right) \in \mathcal{A}_{l} .
$$

We shall abuse notation and will also denote by $\mathcal{A}_{l}$ its image $\Phi_{k}\left(\mathcal{A}_{l}\right) \subset \mathcal{A}_{n}$. Note that $\Phi_{k}\left(\mathcal{A}_{l}^{0}\right) \subset \mathcal{A}_{n}^{0}$ and we also denote this image by $\mathcal{A}_{l}^{0}$.

Given two positive integers $n_{1}, n_{2}$ such that $n_{1}+n_{2}=n$, we introduce the map

$$
\Phi_{n_{1}, n_{2}}: \mathcal{A}_{n_{1}} \times \mathcal{A}_{n_{2}} \longrightarrow \mathcal{A}_{n}
$$

with $a=\Phi_{n_{1}, n_{2}}(b, c)$ defined by the relation $s_{a}=s_{b} \cdot s_{c}$, where $s_{b}=t^{n_{1}}+b_{1} t^{n_{1}-1}+$ $\cdots+b_{n_{1}}$ and $s_{c}=t^{n_{2}}+c_{1} t^{n_{2}-1}+\cdots+c_{n_{2}}$ for $b=\left(b_{1}, \ldots, b_{n_{1}}\right) \in \mathcal{A}_{n_{1}}$ and $c=\left(c_{1}, \ldots, c_{n_{2}}\right) \in \mathcal{A}_{n_{2}}$. We define $\left(\mathcal{A}_{n_{1}} \times \mathcal{A}_{n_{2}}\right)_{0} \subset \mathcal{A}_{n_{1}} \times \mathcal{A}_{n_{2}}$ to be the subset of pairs $(b, c)$ satisfying the relation $b_{1}+c_{1}=0$. We shall denote by $\mathcal{A}_{n_{1}, n_{2}} \subset \mathcal{A}_{n}$ the image of $\Phi_{n_{1}, n_{2}}$ and by $\mathcal{A}_{n_{1}, n_{2}}^{0}$ the subset $\mathcal{A}_{n_{1}, n_{2}} \cap \mathcal{A}_{n}^{0}=\Phi_{n_{1}, n_{2}}\left[\left(\mathcal{A}_{n_{1}} \times \mathcal{A}_{n_{2}}\right)_{0}\right]$.

\subsection{Proof of Theorem 1.2}

We show here the analogue of Theorem 1.2 for the GL(n)-Hitchin space $\mathcal{A}_{n}$. Note that both statements are equivalent by Remark 2.5. Given a spectral cover $\pi: X_{a} \rightarrow C$ with $a \in \mathcal{A}_{n}$, we denote the subgroup of $\operatorname{Pic}^{0}(C)$ defined in (2) by $K_{a}$. Let $\Gamma \subset \operatorname{Pic}^{0}(C)[n]$ be a cyclic subgroup of order $d$. 
Theorem 5.3 We have an equivalence

$$
\Gamma \subset K_{a} \quad \Longleftrightarrow \quad a \in \mathcal{A}_{\Gamma} .
$$

Proof We first show the equivalence in the case the spectral cover $X_{a}$ is integral. In that case we can consider its normalization $\tilde{X}_{a}$, which comes with a natural projection

$$
\tilde{\pi}_{a}: \tilde{X}_{a} \longrightarrow C \text {. }
$$

By Birkenhake and Lange [4, Proposition 11.4.3] we have $\Gamma \subset K_{a}$ if and only if $\tilde{\pi}_{a}$ factors through the map $\varphi$, that is, there exists a map $u: \tilde{X}_{a} \rightarrow D$ such that $\tilde{\pi}_{a}=\varphi \circ u$. By the universal property of the fiber product there exists a map $\delta: \tilde{X}_{a} \rightarrow Y_{a}$ into the fiber product $Y_{a}$ of $X_{a}$ with $D$ over $C$. We denote by $Z=\operatorname{im}(\delta) \subset Y_{a}$ the image of the smooth irreducible curve $\tilde{X}_{a}$. Then $Z$ is irreducible too. Moreover, since $X_{a}$ is reduced and $\varphi$ is étale, the curve $Y_{a}$ is also reduced, hence $Z$ is integral. The group $\Gamma$ acts on $Y_{a}$, hence permutes its irreducible components. Since $\Gamma$ acts transitively on the fibers of $Y_{a} \rightarrow X_{a}$, all irreducible components are of the form $Z^{\sigma}$ for some $\sigma \in \Gamma$. We therefore obtain a factorization $\tilde{X}_{a} \rightarrow Z \rightarrow X_{a}$. Since this composite map is birational, we deduce that $\operatorname{deg}\left(Z / X_{a}\right)=1$. Hence, since $\operatorname{deg}\left(Y_{a} / X_{a}\right)=d$, we conclude that

$$
Y_{a}=\bigcup_{\sigma \in \Gamma} Z^{\sigma} \quad \text { and } \quad Z^{\sigma} \neq Z^{\sigma^{\prime}} \text { if } \sigma \neq \sigma^{\prime} .
$$

By Lemma 2.4 the curve $Z$ is a spectral cover of degree $m$ over $D$ and by Lemma 5.2 we obtain that $a \in \mathcal{A}_{\Gamma}$.

Conversely, for $a \in \mathcal{A}_{\Gamma}$ the map $Z \rightarrow X_{a}$ given by Lemma 5.2 is birational. Hence the normalization of $Z$ equals $\tilde{X}_{a}$ and we obtain a factorization $\tilde{X}_{a} \rightarrow Z \rightarrow D \rightarrow C$, which implies that $\Gamma \subset K_{a}$ by [4, Proposition 11.4.3].

Now we will prove the equivalence for more general characteristics $a \in \mathcal{A}_{n}$. We start with $a \in \mathcal{A}_{n}$ such that the spectral cover $X_{a}$ is irreducible, but not reduced. Let $X_{a}^{\text {red }}$ be the underlying reduced curve of $X_{a}$ and let $k$ be the multiplicity of $X_{a}^{\text {red }}$ in $X_{a}$. We put $n=k \cdot l$. By Lemma 2.4 we have $X_{a}^{\text {red }}=X_{a_{\text {red }}}$ for some characteristic $a_{\text {red }} \in \mathcal{A}_{l} \subset \mathcal{A}_{n}$ and $a=\Phi_{k}\left(a_{\text {red }}\right)-$ see Section 2.2. Then by formula (2) we have $K_{a}=[k]^{-1}\left(K_{a_{\text {red }}}\right)$. We introduce $\Gamma_{\text {red }}=[k](\Gamma) \subset \operatorname{Pic}^{0}(C)[l]$. Then $\Gamma_{\text {red }}$ is a cyclic subgroup of order $d_{\text {red }}=\frac{d}{\operatorname{gcd}(k, d)}$. With this notation we easily obtain the equivalence

$$
\Gamma \subset K_{a} \quad \Longleftrightarrow \quad \Gamma_{\text {red }} \subset K_{a_{\text {red }}} .
$$

We combine this equivalence with the statement of the Theorem written for the integral characteristic $a_{\text {red }}$, which was proved above:

$$
\Gamma_{\text {red }} \subset K_{a_{\text {red }}} \quad \Longleftrightarrow \quad a_{\text {red }} \in \mathcal{A}_{\Gamma_{\text {red }}} \text {. }
$$


Therefore it remains to show the following equivalence

$$
a_{\text {red }} \in \mathcal{A}_{\Gamma_{\text {red }}} \quad \Longleftrightarrow \quad a=\Phi_{k}\left(a_{\text {red }}\right) \in \mathcal{A}_{\Gamma} .
$$

In order to show this equivalence we introduce the subgroup $S=\operatorname{ker}\left(\Gamma \rightarrow \Gamma_{\text {red }}\right)$. By Galois theory there exists an intermediate cover $D \rightarrow \bar{D} \rightarrow C$ with $\operatorname{Aut}(\bar{D} / C)=\Gamma_{\text {red }}$ and $\operatorname{Aut}(D / \bar{D})=S$.

Consider a characteristic $a_{\text {red }} \in \mathcal{A}_{\Gamma_{\text {red }}}$. By Lemma 5.2 applied to $a_{\text {red }} \in \mathcal{A}_{\Gamma_{\text {red }}}$ we obtain that the fiber product $Y_{a_{\text {red }}}=X_{a_{\text {red }} \times} \times \bar{D}$ decomposes as $\bigcup_{\sigma \in \Gamma_{\text {red }}} W^{\sigma}$, where $W$ is a spectral cover of degree $\frac{l}{d_{\text {red }}}$ over $\bar{D}$. Now, observing that $Y_{a}=k\left(Y_{a_{\mathrm{red}}} \times{ }_{\bar{D}} D\right)$ as divisors in $|N|$, we can write

$$
Y_{a}=k \bigcup_{\sigma \in \Gamma_{\text {red }}}\left(W \times_{\bar{D}} D\right)^{\sigma}=\bigcup_{\sigma \in \Gamma} Z^{\sigma},
$$

where we have put $Z=\frac{k}{\operatorname{gcd}(k, d)}\left(W \times{ }_{\bar{D}} D\right) \subset|N|$. Note that $Z^{\sigma}=Z$ for $\sigma \in S$ and that $Z$ is a spectral cover of degree $\frac{n}{d}$. This proves that $a=\Phi_{k}\left(a_{\text {red }}\right) \in \mathcal{A}_{\Gamma}$.

Conversely, we consider a characteristic $a_{\text {red }} \in \mathcal{A}_{l}$ with $\Phi_{k}\left(a_{\text {red }}\right) \in \mathcal{A}_{\Gamma}$. We assume that the spectral cover $X_{a_{\text {red }}}$ is integral. This assumption implies that the fiber product $X_{a_{\mathrm{red}}} \times_{C} D$ is reduced. Let $\mathcal{I}$ denote an irreducible component of $X_{a_{\mathrm{red}}} \times_{C} D$, let $\operatorname{Stab}(\mathcal{I})$ denote its stabilizer, that is,

$$
\operatorname{Stab}(\mathcal{I})=\left\{\sigma \in \Gamma \mid \mathcal{I}^{\sigma}=\mathcal{I}\right\},
$$

and let $\delta=|\operatorname{Stab}(\mathcal{I})|$ be the order. Since $\Gamma$ acts transitively on the fibers of $X_{a_{\mathrm{red}} \times{ }_{C}} D \rightarrow$ $X_{a_{\text {red }}}$ we obtain the decomposition into irreducible components

$$
X_{a_{\mathrm{red}}} \times_{C} D=\bigcup_{\sigma \in \Gamma / \operatorname{Stab}(\mathcal{I})} \mathcal{I}^{\sigma}
$$

Let us denote by $s$ the global section over $|N|$ with $\operatorname{Zeros}(s)=\mathcal{I}$. Then the spectral cover $Y_{a}=k\left(X_{a_{\mathrm{red}}} \times_{C} D\right)$ is the zero set of the section

$$
\prod_{\sigma \in \Gamma / \operatorname{Stab}(\mathcal{I})}\left(s^{\sigma}\right)^{k},
$$

which has $k \frac{d}{\delta}$ irreducible factors of the same degree. The assumption $\Phi_{k}\left(a_{\text {red }}\right) \in \mathcal{A}_{\Gamma}$ implies that this product can be written as a product of $d$ factors of the same degree, hence $k \frac{d}{\delta}$ is divisible by $d$, that is, $\delta$ divides $k$, so $\delta$ divides $\operatorname{gcd}(k, d)$. Since $\delta=|\operatorname{Stab}(\mathcal{I})|$ and $\operatorname{gcd}(k, d)=|S|$, we conclude that $\operatorname{Stab}(\mathcal{I}) \subset S$. We then introduce the section

$$
t=\prod_{\sigma \in S / \operatorname{Stab}(\mathcal{I})} s^{\sigma}
$$


Since $t$ is $S$-invariant, its zero divisor descends as a spectral cover $W$ over $\bar{D}=D / S$. Moreover we have the equality

$$
\prod_{\sigma \in \Gamma / \operatorname{Stab}(\mathcal{I})} s^{\sigma}=\prod_{\tau \in \Gamma_{\mathrm{red}}=\Gamma / S} t^{\tau},
$$

which proves that $Y_{a_{\text {red }}}=X_{a_{\text {red }}} \times_{C} \bar{D}$ splits into $d_{\text {red }}$ spectral covers $W^{\tau}$ for $\tau \in \Gamma_{\text {red }}$, and we conclude by Lemma 5.2 that $a_{\text {red }} \in \mathcal{A}_{\Gamma_{\text {red }}}$.

Finally, we will show the equivalence for a characteristic $a \in \mathcal{A}_{n_{1}, n_{2}} \subset \mathcal{A}_{n}$, that is, the spectral cover $X_{a}$ equals the union $X_{a_{1}} \cup X_{a_{2}}$ for two spectral covers $X_{a_{i}}$ with $a_{i} \in \mathcal{A}_{n_{i}}$, which we assume to be irreducible. Then by (2) we have

$$
\Gamma \subset K_{a} \quad \Longleftrightarrow \quad \Gamma \subset K_{a_{1}} \text { and } \Gamma \subset K_{a_{2}} \text {. }
$$

On the other hand since the curves $X_{a_{i}}$ are irreducible, we can apply what we have proved above, that is, for $i=1,2$

$$
\Gamma \subset K_{a_{i}} \quad \Longleftrightarrow \quad a_{i} \in \mathcal{A}_{\Gamma}^{n_{i}} .
$$

Note that $\Gamma \subset \operatorname{Pic}^{0}(C)\left[n_{i}\right]$ for $i=1,2$. Here $\mathcal{A}_{\Gamma}^{n_{i}}$ denotes the corresponding subspace of $\mathcal{A}_{n_{i}}$. Hence it remains to show that

$$
a_{1} \in \mathcal{A}_{\Gamma}^{n_{1}} \text { and } a_{2} \in \mathcal{A}_{\Gamma}^{n_{2}} \quad \Longleftrightarrow \quad a=\Phi_{n_{1}, n_{2}}\left(a_{1}, a_{2}\right) \in \mathcal{A}_{\Gamma} .
$$

The implication $\Rightarrow$ is trivial. In order to show the implication $\Leftarrow$ we note that Lemma 5.2 gives the decomposition $Y_{a}=\bigcup_{\sigma \in \Gamma} Z^{\sigma}$ for some spectral cover $Z$. We then put $Z_{i}=Z \cap Y_{a_{i}}$, which gives the desired decomposition for the fiber product $Y_{a_{i}}$.

Now the statement follows for arbitrary characteristic $a \in \mathcal{A}_{n}$ by induction on the number of irreducible components of $X_{a}$.

\section{Moduli space of semi-stable Higgs bundles}

In this section we describe how the $\operatorname{Prym}$ variety $\operatorname{Prym}\left(X_{a} / C\right)$ acts on the fiber $h^{-1}(a)$ of the $\mathrm{SL}_{n}$-Hitchin fibration. First we recall the semi-stability condition Definition 3.7 for coherent sheaves over the spectral cover $X_{a}$. The following result generalizes Beauville, Narasimhan and Ramanan [3, Proposition 3.6] to any spectral cover $X_{a}$.

Proposition 6.1 Let $a \in \mathcal{A}_{n}^{0}$ be any characteristic. The fiber $h^{-1}(a)$ of the $\mathrm{SL}_{n}-$ Hitchin fibration (1) equals the moduli space of semi-stable sheaves $\mathcal{E}$ over the spectral curve $X_{a}$ of rank 1 and with fixed determinant $\operatorname{det}\left(\pi_{*} \mathcal{E}\right)=\Delta$. 
Proof In the case $X_{a}$ integral this is exactly [3, Proposition 3.6] except for the assertion that the bijective correspondence between Higgs bundles $(E, \phi)$ over $C$ with given characteristic $a$ and torsion free rank- 1 sheaves $\mathcal{E}$ over $X_{a}$ is compatible with both semi-stability conditions. But this compatibility is shown by Simpson [32, Corollary 6.9] — note that Simpson works in the special case when $M$ is the canonical bundle of $C$ but his proof remains valid for an arbitrary line bundle $M$.

In order to show the statement for an arbitrary spectral cover $X_{a}$ we observe that the constructions and arguments of Beauville, Narasimhan and Ramanan [3] and Simpson [32] remain valid for an arbitrary characteristic $a$. However the next statement needs a new proof.

Lemma 6.2 Let $\mathcal{E}$ be a torsion-free sheaf over $X_{a}$. Then the characteristic of its associated Higgs bundle $(E, \phi)$ equals $a$.

Proof If $X_{a}$ is integral, this is shown in [3, Proposition 3.6] using the irreducibility of the polynomial $s_{a}$ defined in (6). In the general case we first show the statement when $X_{a}$ is irreducible but non-reduced and secondly when $X_{a}$ is reducible. Given a sheaf $\mathcal{E}$ over $X_{a}$ we denote by $\operatorname{Char}(\mathcal{E})$ the characteristic polynomial of its associated Higgs field.

Suppose first that $X_{a}$ is irreducible and that its underlying reduced curve $X_{a}^{\text {red }}$ has multiplicity $m$. Denote by $\mathcal{E}^{\text {red }}$ the restriction of $\mathcal{E}$ to $X_{a}^{\text {red }}$. Then one shows exactly along the lines of Lemma 3.5 that $\operatorname{Char}(\mathcal{E})=\operatorname{Char}\left(\mathcal{E}^{\text {red }}\right)^{m}$.

Suppose now that $X_{a}$ has several irreducible components $X_{i}$ and denote by $\mathcal{E}_{i}$ the restriction of $\mathcal{E}$ to $X_{i}$. Then one shows exactly along the lines of Lemma 3.6 that $\operatorname{Char}(\mathcal{E})=\prod_{i} \operatorname{Char}\left(\mathcal{E}_{i}\right)$.

Finally decomposing $X_{a}$ into irreducible components and taking the underlying reduced curves, one reduces the statement to the integral case, which is already shown.

This completes the proof of the proposition.

Considering $h^{-1}(a)$ as the moduli space of semi-stable sheaves over $X_{a}$ we let $\operatorname{Prym}\left(X_{a} / C\right)$ act through tensor product. By Proposition 3.10 it is clear that the determinant is invariant under this action, and since $\operatorname{deg}(\mathcal{E} \otimes \mathcal{L})=\operatorname{deg}(\mathcal{E})$ for any $\mathcal{L} \in \operatorname{Prym}\left(X_{a} / C\right)$ we see that semi-stability is preserved.

Remark 6.3 In a forthcoming paper we will describe in detail the case $a=0$, that is, the action of the Prym variety $\operatorname{Prym}\left(X_{0} / C\right)$, which equals in this case $n^{2 g}$ copies of a vector space (see, for example, Drézet [7, Section 3.3]), on the nilpotent cone of the Higgs moduli space. 
Remark 6.4 We note that the description of the fiber $h^{-1}(a)$ above was already stated by Schaub [30, Proposition 2.1]. Unfortunately the proof of [30, Proposition 2.1] contains an inaccuracy, which is a consequence of the author's different definition of a torsion-free sheaf of rank 1 over an arbitrary spectral cover.

\section{Application to Topological Mirror Symmetry}

In this final section we assume that our line bundle $M$ equals the canonical bundle $K_{C}$ and that $(n, \operatorname{deg}(\Delta))=1$. These assumptions imply that $\mathcal{M}$ is a non-singular quasi-projective variety of dimension

$$
\operatorname{dim}(\mathcal{M})=\left(n^{2}-1\right)(2 g-2) .
$$

The dimension of the affine space $\mathcal{A}_{n}^{0}$ is

$$
\operatorname{dim}\left(\mathcal{A}_{n}^{0}\right)=\left(n^{2}-1\right)(g-1)
$$

and consequently the Hitchin map $h$ is of relative dimension $\left(n^{2}-1\right)(g-1)$.

Let $\Gamma \subset \operatorname{Pic}^{0}(C)[n]$ be a cyclic subgroup of order $d$ which must divide $n$. Then we have the following:

Lemma 7.1 $\operatorname{dim}\left(\mathcal{A}_{\Gamma}^{0}\right)=\left(n^{2} / d-1\right)(g-1)$

Proof In Section 5.1 we associated an étale Galois cover $\phi: D \rightarrow C$ to $\Gamma$ with Galois group $\Gamma$. Thus the pull back $N=\phi^{*}\left(K_{C}\right)=K_{D}$. There we also constructed $\mathcal{A}_{\Gamma}^{0}$ as the image of $\mathcal{A}_{m}^{\Gamma}$ by the finite map $\Phi_{\Gamma}$. Thus we have

$$
\begin{aligned}
\operatorname{dim}\left(\mathcal{A}_{\Gamma}^{0}\right) & =\operatorname{dim}\left(\mathcal{A}_{m}^{\Gamma}\right) \\
& =\operatorname{dim}\left(\mathcal{A}_{m}\right)-\operatorname{dim}\left(H^{0}\left(D, K_{D}\right)^{\Gamma}\right) \\
& =\operatorname{dim}\left(\mathcal{A}_{m}\right)-\operatorname{dim}\left(H^{0}\left(C, K_{C}\right)\right) \\
& =\frac{n^{2}}{d^{2}}(d g-d)+1-g \\
& =\left(n^{2} / d-1\right)(g-1) .
\end{aligned}
$$

as required.

Now we can prove Theorem 1.4: 
Proof of Theorem 1.4 With the notation (3) we introduce

$$
\mathcal{A}_{n e}^{0}:=\mathcal{A}_{n}^{0} \backslash \mathcal{A}_{\text {endo }}
$$

the locus of those characteristics for which $\operatorname{Prym}\left(X_{a} / C\right)$ is connected. Further denote

$$
\mathcal{M}_{n e}:=h^{-1}\left(\mathcal{A}_{n e}^{0}\right) \text {. }
$$

First we argue that $\operatorname{Pic}^{0}(C)[n]$ acts trivially on $H^{*}\left(\mathcal{M}_{n e}\right)$. Let $a \in \mathcal{A}_{n e}^{0}$. Since by (8) we have $\pi^{*} \operatorname{Pic}^{0}(C)[n] \subset \operatorname{Prym}\left(X_{a} / C\right)$ and since by assumption on the characteristic $a$ the Prym variety $\operatorname{Prym}\left(X_{a} / C\right)$ is connected, the finite group $\operatorname{Pic}^{0}(C)[n]$ acts trivially on the cohomology of every fiber $h_{n e}^{-1}(a)$ of

$$
h_{n e}:=\left.h\right|_{\mathcal{M}_{n e}}: \mathcal{M}_{n e} \rightarrow \mathcal{A}_{n e}^{0} .
$$

Therefore by Lemma 7.2 below it follows that it acts trivially on $H^{*}\left(\mathcal{M}_{n e} ; \mathbb{Q}\right)$ as well.

Lemma 7.2 Let $f: X \rightarrow Y$ be a proper map between locally compact Hausdorff topological spaces. Let the finite group $\mathrm{G}$ act on $X$ along the fibers of $f$. Assume $\mathrm{G}$ acts trivially on $H^{*}\left(X_{a} ; \mathbb{Q}\right)$ for every $a \in Y$, where $X_{a}=f^{-1}(a)$. Then the action of $\mathrm{G}$ on $H^{*}(X ; \mathbb{Q})$ is trivial.

Proof Let $\mathbb{Q}_{X}$ be the constant sheaf on $X$. Let $\pi: X \rightarrow X / \mathrm{G}$ be the quotient map, $\mathbb{Q} X / \mathrm{G}$ the constant sheaf on $X / \mathrm{G}$ and $g: X / \mathrm{G} \rightarrow Y$ defined by the property $g \circ \pi=f$. Then we have a morphism of sheaves

$$
m: \underline{\mathbb{Q}} x / \mathrm{G} \rightarrow \pi_{*} \underline{\mathbb{Q}} X .
$$

This induces a morphism of sheaves

$$
R^{k} m: R^{k} g_{*} \mathbb{Q}_{X / \mathrm{G}} \rightarrow R^{k} g_{*} \pi_{*} \underline{\mathbb{Q}}_{X} .
$$

As $\pi$ is finite $R^{k} g_{*} \pi_{*} \underline{\mathbb{Q}}_{X} \cong R^{k} f_{*} \underline{\mathbb{Q}}_{X}$. Thus we get a morphism of sheaves

$$
R^{k} m: R^{k} g_{*} \underline{\mathbb{Q}}_{X / \mathrm{G}} \rightarrow R^{k} f_{*} \underline{\mathbb{Q}}_{X} .
$$

Firs we understand the induced map on any stalk. By proper base change (see Iversen [19, Theorem 6.2]) the stalks are just the $k$ th cohomology groups of the fibres. Thus our morphism becomes

$$
R^{k} m_{s}: H^{k}\left(X_{s} / \mathrm{G} ; \mathbb{Q}\right) \rightarrow H^{k}\left(X_{s} ; \mathbb{Q}\right) .
$$

We have the isomorphism

$$
H^{k}\left(X_{s} / \mathrm{G} ; \mathbb{Q}\right) \cong H^{k}\left(X_{s} ; \mathbb{Q}\right)^{\mathrm{G}}
$$


of Macdonald [24, (1.2)] and by assumption

$$
H^{k}\left(X_{s} ; \mathbb{Q}\right)^{\mathrm{G}} \cong H^{k}\left(X_{s} ; \mathbb{Q}\right) .
$$

Thus $R^{k} m_{s}$ is an isomorphism for all $s \in Y$ so Hartshorne [14, Proposition 1.1] implies that $R^{k} m$ is an isomorphism of sheaves. By [24, (1.2)] the theorem follows.

Thus we have that $\operatorname{Pic}^{0}(C)[n]$ acts trivially on $H^{*}\left(\mathcal{M}_{n e} ; \mathbb{Q}\right)$. Note that by Lemma 7.1 and by the observation that $\mathcal{A}_{\Gamma_{2}}^{0} \subset \mathcal{A}_{\Gamma_{1}}^{0}$ if $\Gamma_{1} \subset \Gamma_{2}$, the codimension of the endoscopic locus $\mathcal{A}_{\text {endo }}$ is given by

$$
c_{n}:=\left(n^{2}-1\right)(g-1)-\left(n^{2} / p_{n}-1\right)(g-1)=n^{2}\left(1-1 / p_{n}\right)(g-1),
$$

where $p_{n}$ is the smallest prime divisor of $n$. By studying the cohomology long exact sequence of the pair $\left(\mathcal{M}, \mathcal{M}_{n e}\right)$ we see that the restriction map

$$
H^{k}(\mathcal{M} ; \mathbb{Q}) \rightarrow H^{k}\left(\mathcal{M}_{n e} ; \mathbb{Q}\right)
$$

is an isomorphism for $k \leq 2 c_{n}-2$ and is an injection for $k=2 c_{n}-1$. Thus we could immediately deduce that $\operatorname{Pic}^{0}(C)[n]$ acts trivially on $H^{k}(\mathcal{M} ; \mathbb{Q})$ for

$$
k<2 c_{n} .
$$

Further, we note that any generic $\left(c_{n}-1\right)$-dimensional subvariety $\Lambda^{c_{n}-1}$ of $\mathcal{A}^{0}$ will be disjoint from $\cup \mathcal{A}_{\Gamma}^{0}$ thus a cohomology class $\eta \in H^{*}(\mathcal{M} ; \mathbb{Q})$ which is not invariant under $\operatorname{Pic}^{0}(C)[n]$ (we call such classes variant) must satisfy

$$
\left.\eta\right|_{h^{-1}\left(\Lambda^{c_{n}-1}\right)}=0
$$

and so by de Cataldo, Hausel and Migliorini [6, Theorem 1.4.8] a variant class of degree $i$ must have perversity at most $i-c_{n}$. By [6, Theorem 1.4.12] this already implies $i \geq 2 c_{n}$ (which as we noted above in (12) also follows from the cohomology long exact sequence of $\left.\left(\mathcal{M}, \mathcal{M}_{n e}\right)\right)$. Finally a variant class

$$
\eta \in H_{c_{n}}^{2 c_{n}}(\mathcal{M} ; \mathbb{Q})
$$

by Relative Hard Lefschetz -with the ample $\operatorname{Pic}^{0}(C)[n]$-invariant class $\alpha \in H^{2}(\mathcal{M} ; \mathbb{Q})$ gives a variant class

$$
\alpha^{\operatorname{dim}(\mathcal{M}) / 2-c_{n}} \eta \in H^{\operatorname{dim}(\mathcal{M})}(\mathcal{M} ; \mathbb{Q})
$$

which contradicts García-Prada, Heinloth and Schmitt [10, Theorem 1], which proves that there are no variant classes in the middle cohomology $H^{\operatorname{dim}(\mathcal{M})}(\mathcal{M} ; \mathbb{Q})$. Here we used the fact $\alpha \in H^{2}(\mathcal{M} ; \mathbb{Q})$ is always $\operatorname{Pic}^{0}(C)[n]$-invariant, for example because of (12) and $2<2 c_{n}$ when $g>1$. 
Thus the smallest possible degree a variant class may have is $2 c_{n}+1$, proving Theorem 1.4.

Remark 7.3 Theorem 1.4 is a consequence of the topological mirror symmetry conjecture (see Hausel and Thaddeus [17, Conjecture 5.1]). It can be deduced by calculating all possible fermionic shifts $F(\gamma)$ for $\gamma \in \operatorname{Pic}^{0}(C)[n]$ on the RHS of [17, Conjecture 5.1] and noting that due to the presence of the gerbe $\widehat{B}^{d}$ the degree 0 part of the cohomology of the twisted sector $\mathcal{M}^{\gamma} / \mathrm{Pic}^{0}(C)[n]$ does not contribute to the stringy cohomology of $\mathcal{M} / \operatorname{Pic}^{0}(C)[n]$. In fact, the inequality in Theorem 1.4 is sharp, when $n$ is a prime, because [17, Proposition 10.1] implies that there is variant cohomology in degree $2 c_{n}+1$. The close connection between the topological mirror symmetry conjecture [17, Conjecture 5.1] and the group of connected components of Prym varieties of spectral covers unravelled in this section is an indication of the deep analogies between S-duality considerations in physics as in Kapustin and Witten's [20] and Ngô's [27; 28] geometric approach to the fundamental lemma. For more discussion on this see Hausel [15].

Finally we can prove Corollary 1.5:

Proof of Corollary 1.5 As the Hitchin map $h: \mathcal{M} \rightarrow \mathcal{A}_{n}^{0}$ is proper by Nitsure [29], and the $\mathbb{C}^{\times}$-action on $\mathcal{M}$ covers a $\mathbb{C}^{\times}$action on $\mathcal{A}_{n}^{0}$ with positive weights, and so with a unique fixed point $0 \in \mathcal{A}^{0}$. It follows that for every $z \in \mathcal{M}$ the $\operatorname{limit}_{\lambda \rightarrow 0} \lambda z$ exists in the compact fixed point set $\mathcal{M}^{\mathbb{C}^{\times}}$. Using the compactification $\overline{\mathcal{M}}$ as defined in such a situation in Simpson [33, Section 11] and Hausel [16] we can apply the following cohomological techniques. They were studied in the compact case by Kirwan in [21], (who commented on the non-compact case in [21, Section 9]) and in the non-compact case by Nakajima in [25, Section 5.1].

For a connected component $F_{\alpha}$ of the fixed point set

$$
\mathcal{M}^{\mathbb{C}^{\times}}=\bigsqcup_{\alpha \in I} F_{\alpha}
$$

we can then define

$$
U_{\alpha}:=\left\{z \in \mathcal{M} \mid \lim _{\lambda \rightarrow 0} \lambda z \in F_{\alpha}\right\}
$$

giving the Bialinicki-Birula decomposition

$$
\mathcal{M}=\bigsqcup_{\alpha \in I} U_{\alpha}
$$


One can now define a partial ordering on the index set $I$, by $\alpha \leq \beta$ when $U_{\beta} \cap \bar{U}_{\alpha} \neq \varnothing$. By induction on this ordering one can prove

$$
H^{*}(\mathcal{M} ; \mathbb{Q}) \cong \bigoplus_{\alpha \in I} H^{*-\operatorname{codim}\left(U_{\alpha}\right)}\left(U_{\alpha} ; \mathbb{Q}\right)
$$

If 0 denotes the minimal element in $I$, then we can see that $U_{0}=T^{*} \mathcal{N}$ contains those Higgs bundles where the underlying bundle is stable. It follows from (13) (or rather via the perfectness of the Bialinicki-Birula stratification used to prove (13)) that the restriction map

$$
H^{*}(\mathcal{M} ; \mathbb{Q}) \rightarrow H^{*}\left(U_{0} ; \mathbb{Q}\right) \cong H^{*}(\mathcal{N} ; \mathbb{Q})
$$

is surjective.

Thus in particular Theorem 1.4 implies that $\operatorname{Pic}^{0}(C)[n]$ acts trivially on the cohomology $H^{k}(\mathcal{N} ; \mathbb{Q})$ for $k \leq 2 c_{n}=2 n^{2}\left(1-1 / p_{n}\right)(g-1)$, where $p_{n}$ is the smallest prime divisor of $n$. But

$$
2 c_{n}>\left(n^{2}-1\right)(g-1)
$$

thus the cohomology $H^{*}(\mathcal{N} ; \mathbb{Q})$ is $\operatorname{Pic}^{0}(C)[n]$-invariant up to and including the middle degree and by Poincaré duality everywhere. The result follows.

As $2 c_{n}-1 \geq\left(n^{2}-1\right)(g-1)$ (with possible equality when $g=2$ and $p_{n}=2$ ) we note that the more elementary estimate (12) already implies the result.

Remark 7.4 When $n=2$ Corollary 1.5 follows from Newstead [26, Theorem 1] and for general $n$ it appeared as [13, Theorem 1] of Harder-Narasimhan. In [13] it was proved by an arithmetic study of $\mathcal{N}$. Later it was reproved with the gauge theoretical approach of Atiyah-Bott in [2, Proposition 9.7]. Our proof above is by identifying the characteristics for which the Prym variety is connected, which excludes the nilpotent cone, where $\mathcal{N}$ is located, and yields Corollary 1.5 without focusing on $H^{*}(\mathcal{N} ; \mathbb{Q})$. This approach thus can be considered as an example of the abelianization philosophy of Atiyah-Hitchin [1, Section 6.3], by studying the abelian problem of Prym varieties of spectral curves we deduced results on the moduli space $\mathcal{N}$ of vector bundles of rank $n>1$.

\section{References}

[1] M Atiyah, The geometry and physics of knots, Lezioni Lincee., Cambridge Univ. Press (1990) MR1078014

[2] MF Atiyah, R Bott, The Yang-Mills equations over Riemann surfaces, Philos. Trans. Roy. Soc. London Ser. A 308 (1983) 523-615 MR702806 
[3] A Beauville, MS Narasimhan, S Ramanan, Spectral curves and the generalised theta divisor, J. Reine Angew. Math. 398 (1989) 169-179 MR998478

[4] C Birkenhake, H Lange, Complex abelian varieties, second edition, Grundl. Math. Wissen. 302, Springer, Berlin (2004) MR2062673

[5] P-H Chaudouard, G Laumon, Le lemme fondamental pondéré II: Énoncés cohomologiques arXiv:0912.4512

[6] M A de Cataldo, T Hausel, L Migliorini, Topology of Hitchin systems and Hodge theory of character varieties: the case $A_{1}$, Ann. of Math. 175 (2012) 1329-1407

[7] J-M Drézet, Faisceaux cohérents sur les courbes multiples, Collect. Math. 57 (2006) 121-171 MR2223850

[8] J-M Drézet, Faisceaux sans torsion et faisceaux quasi localement libres sur les courbes multiples primitives, Math. Nachr. 282 (2009) 919-952 MR2541242

[9] E Frenkel, E Witten, Geometric endoscopy and mirror symmetry, Commun. Number Theory Phys. 2 (2008) 113-283 MR2417848

[10] O García-Prada, J Heinloth, A Schmitt, On the motives of moduli of chains and Higgs bundles arXiv:1104.5558

[11] A Grothendieck, Éléments de géométrie algébrique II: Étude globale élémentaire de quelques classes de morphismes, Publ. Math. Inst. Hautes Etudes Sci. 8 (1961) 5-205

[12] A Grothendieck, Éléments de géométrie algébrique IV: Étude locale des schémas et des morphismes de schémas, Publ. Math. Inst. Hautes Etudes Sci. (1967) 5-333 MR0238860

[13] G Harder, MS Narasimhan, On the cohomology groups of moduli spaces of vector bundles on curves, Math. Ann. 212 (1974/75) 215-248 MR0364254

[14] R Hartshorne, Algebraic geometry, Graduate Texts in Mathematics 52, Springer, New York (1977) MR0463157

[15] T Hausel, Global topology of the Hitchin system arXiv:1102.1717

[16] T Hausel, Compactification of moduli of Higgs bundles, J. Reine Angew. Math. 503 (1998) 169-192 MR1650276

[17] T Hausel, M Thaddeus, Mirror symmetry, Langlands duality, and the Hitchin system, Invent. Math. 153 (2003) 197-229 MR1990670

[18] N Hitchin, Stable bundles and integrable systems, Duke Math. J. 54 (1987) 91-114 MR885778

[19] B Iversen, Cohomology of sheaves, Universitext, Springer, Berlin (1986) MR842190

[20] A Kapustin, E Witten, Electric-magnetic duality and the geometric Langlands program, Commun. Number Theory Phys. 1 (2007) 1-236 MR2306566

[21] F C Kirwan, Cohomology of quotients in symplectic and algebraic geometry, Mathematical Notes 31, Princeton Univ. Press (1984) MR766741 
[22] S L Kleiman, The Picard scheme, from: "Fundamental algebraic geometry", Math. Surveys Monogr. 123, Amer. Math. Soc., Providence, RI (2005) 235-321 MR2223410

[23] Q Liu, Algebraic geometry and arithmetic curves, Oxford Graduate Texts in Mathematics 6, Oxford University Press (2002) MR1917232 Translated from the French by Reinie Erné, Oxford Science Publications

[24] I G Macdonald, Symmetric products of an algebraic curve, Topology 1 (1962) 319-343 MR0151460

[25] H Nakajima, Lectures on Hilbert schemes of points on surfaces, University Lecture Series 18, American Mathematical Society (1999) MR1711344

[26] P E Newstead, Characteristic classes of stable bundles of rank 2 over an algebraic curve, Trans. Amer. Math. Soc. 169 (1972) 337-345 MR0316452

[27] B C Ngô, Fibration de Hitchin et endoscopie, Invent. Math. 164 (2006) 399-453 MR2218781

[28] B C Ngôo, Le lemme fondamental pour les algèbres de Lie, Publ. Math. Inst. Hautes Études Sci. (2010) 1-169 MR2653248

[29] N Nitsure, Moduli space of semistable pairs on a curve, Proc. London Math. Soc. 62 (1991) 275-300 MR1085642

[30] D Schaub, Courbes spectrales et compactifications de jacobiennes, Math. Z. 227 (1998) 295-312 MR1609069

[31] C T Simpson, Moduli of representations of the fundamental group of a smooth projective variety I, Inst. Hautes Études Sci. Publ. Math. (1994) 47-129 MR1307297

[32] C T Simpson, Moduli of representations of the fundamental group of a smooth projective variety II, Inst. Hautes Études Sci. Publ. Math. (1994) 5-79 MR1320603

[33] C Simpson, The Hodge filtration on nonabelian cohomology, from: "Algebraic geometry-Santa Cruz 1995”, Proc. Sympos. Pure Math. 62, Amer. Math. Soc., Providence, RI (1997) 217-281 MR1492538

Section de Mathématiques, École Polytechnique Fédéral de Lausanne

Section 8, CH-1015 Lausanne, Switzerland

Laboratoire de Mathématiques J.A. Dieudonné, UMR no 7351 CNRS UNSA

Université de Nice Sophia-Antipolis, 06108 Nice Cedex 02, France

tamas.hausel@epfl.ch, pauly@unice.fr

http://geom.epfl.ch/Hausel, http://math.unice.fr/ pauly/

Proposed: Frances Kirwan

Seconded: Jim Bryan, Richard Thomas
Received: 29 June 2011

Accepted: 8 June 2012 